\title{
The Complex Quantum-State of Consciousness
}

\author{
Dr.Narayan Kumar Bhadra \\ L. S. S. S. High School (H.S), North 24-Parganas West Bengal, India
}

\begin{abstract}
We introduce a series of new energy sources $S U(6), S U(12), S U(24), \ldots \ldots \ldots \infty$. We consider an earlier stages of quantum physics of the super unified group $S U(11)$ which created two distinct character's energy group $S U(6)$ \& SU(5)by the symmetry breaking of $S U(11))$ [ $\supset S U(6) \times$ $S U(5) \times U(1)]$ of the 10-dimensional space-time beyond the standard model of physics. The extradimensions are associated with the mental i.e. we consider the individual mind is (partly) an expression of the universal mind. Again the 4-dimensional Einstein's space-time obtained from the symmetry breaking of the Unified Gaussian energy group $S U(5)\left[\supset S U(3) \times S U(2)_{L} \times U(1)\right]$. That earlier stages that means in the Super Unified Gaussian Group SU(11) having quark-like particles together strong forces of $S U(6)$ formed conscious particles that means elements-like of the wave characters and with lepton-like particles by the weak forces of $S U(5)$ [we assumed that in the first stage $S U(6)$ creates strong forces while $S U(5)$ creates weak forces] introduces a new dimensions of physics. In the second stage, the atoms of the material elements of our physical universe constructed by the quarks with the strong forces $S U(3)$, weak forces $S U(2)$, and electrodynamics $U(1)$ with respect to the conscious particles or atom-likelelement-like energy particles of the first stage. The new energy sources $S U(6)$ is responsible for different kinds of intelligence/consciousness within our material/physical universe as well as the elements which produces life with the formation of biological particles etc. with the re-arrangement of the different kinds of simple \& compound chemical elements. Different types of matter elements in our physical universe formed by increasing the number of electrons i.e. atomic numbers and then compound elements are found by the chemical reaction, also which was controlled by $S U(6)$. The energy particles of the stage of $S U(11)$ are always in the form of waves, while particles of the stage of SU(5)maintaining the law of wave-particle duality called entanglement. Thus in our physical/objective universe, the consciousness energies and physical energies are always behaving like entanglement. Consciousness/intelligence frequently changes mainly due to the randomly superposition of the wave functions. In the theory of $S U(11)$, it is possible to change any of 30(thirty) latent energy bosons of $S U(6)$ into any of the 30(thirty) matter energy bosons of $S U(5)$ or vice-versa by the exchange of the J-bosons of $S U(11)$. So, at this stage, by the symmetry breaking of $S U(11)$ created an amount of pre-matter energy/exotic matter fluids by exchanging the J-bosons of SU(11). It is assumed that in the first stage, quark-like particles of $u, d, \ldots .$. etc. are 5-times or otherwise 5different types of each $u$, d....etc. that of the quarks of the unified energy groups of SU(5), the standard model of physics. The strong latent energy groups $S U(6)$ produces different kinds of conscious elements after transferring the exotic matter fluids into ordinary matter fluids and producing biological cells, after then $S U(6)$ responsible for the biological consciousness and the sum of the biological consciousness created an individual identity, a complete individual identity means like a human/a single animal/a single plant etc. as like as an element/compound element/atom/molecule etc., in the large scales a cluster/galaxy/star/planet etc. Thus there exists a dualism of an energy waves produces in first stages with $S U(6)$ and material-like with $S U(5)$. The symmetry breaking of the energy group of $S U(5)$ then belongs to the sub-systems only. By the suitable arrangement of the $u, d, \ldots$ etc. of quark-like particles with $S U(6)$, build different kinds of conscious atom-like elements which produces the basic properties of consciousness of the universe and as well as for live elements including human. Therefore, SU(6) behaves like a programmer created a blue-print of the whole universe/galaxy within the black hole/live body within the DNA etc. and then stays in the neighborhood of the material/biological elements as a field. The creation of different carbon based live bodies etc. and their identities depend on an amount of resultant/sum total consciousness with respect to the material elements of the live bodies where consciousness arises, as require as possible and also depend on the situations \& positions of the place of creation of the live body within the universe [in our earth naturally, human, any animal, plant etc of the carbon based live bodies created within a biological temperature. Thus in another place other than earth within the universe like earth, it may be possible to create live bodies like us or otherwise may create live bodies are based on silicon or other matter (here silicon and carbon belongs to the
\end{abstract}


same group of the periodic table of chemistry) within a require temperature because consciousness might be at some point arise in silicon or other matter]. Those two stages mentioned above may be assumed like the state of vapor \& liquid of the same water i.e. we compared with the character of water which is different from the characters of hydrogen \& oxygen, that means from the symmetry breaking of the super-unified group SU(11), we get two distinct characters identity as consciousness or spirit \& physical matter. In the first stage, the energy waves cannot collapse into visible material-like particles but we can assume as pseudo particle while in the second stage wave function may be collapsed into particles by the superposition of the energy waves. Quark/Quarklike particles produces electrons and also in the neighborhood of a bunch of individual neuron created free electrons by the residue lexcess [according to the requirements of fulfillments] energies which play with electromagnetic forces throughout the inner and outer section of a human body and other live bodies. The energy group SU(6) having five neutral bosons out of 35-bosons, one neutral boson created a strong neutral current by the electromagnetic interaction with $U(1)$ [i.e. in the frame-work $S U(6) \times U(1)$ ] [which was explained at CERN, 'the flavor changing neutral current'] as like as created, the weak neutral current of $S U(2)$ with $U(1)$ in the frame-work of $S U(2) \times U(1)$. We consider an electromagnetic interaction created by $S U(6) \& S U(2)$ with $U(1)$ are also caused for different effectiveness of the consciousness, intelligence, emotions and any kind of electromagnetic interactions etc. \& the movements of biological free electrons throughout the human body or any other live-body or between live and inanimate objects. So we can assume that like God with consciousness of the soul energy of the universe on the first stage created our real material universe of the second stage including all lives called physical stuff and stayed within us till to the back to the stage of the Grand Super Unified Group SU(23) beyond 10-dimensional space-time. The wave information is transmitted from and into the brain by wave resonance. It is also estimated that the future may influence the present. Hence the consciousness or soul energy never diminished and stayed within our atmosphere with pseudo-activeness called spirit stuff.

\section{Introduction}

The human brain and its mental aspects are associated with classical brain physiology and are also part of a quantum physical universe. We consider the whole universe being multidimensional. We consider the universe having $(4+\mathrm{D})$-dimensional Friedmann-Robertson-Walker type universe having complex scale factor $R+i R_{I}$, where $R$ is the scale factor corresponding to the usual 4-dimensional Universe while $\mathrm{iR}_{\mathrm{I}}$ is that of $\mathrm{D}$-dimensional space-time. It was shown mathematically that when $\mathrm{D}=6$ (i.e. $4+6=10$ - dimensions), $\mathrm{R}_{\mathrm{I}}=\mathrm{R}$ and then real/physical universe actually starts. With respect to the quantum theory, it was shown that our universe started with the symmetry breaking of the Gaussian energy group $\mathrm{SU}(11)$. We may consider that the extradimensions ' $\mathrm{D}$ ' are associated with a mental or that of the individual mind is (partly) an expression of a universal mind through holonomic communication with quantum fields. This was proposed by the Pribram 1986, 2011, in his holographic (holonomic) theories. The human brain conceived as an interfacing organ that not only produces mind and consciousness but also receives information. The brain or parts of the brain are conceived as an interference hologram of incoming data and already existing data (a "personal universe") which equivalent to the subject's memory. If properly exposed ("analyzed"), information about the outer world can be distilled where "analyzer" is cerebral electrophysiology. Again "Bohm" hypothesized that additional dimensions (i.e. extra-dimensions) are necessary to describe $\mathrm{QM}$ interference processes, thereby circumventing probabilistic theories and consciousness-induced collapse of the wave function. According to this theory, assumed that the universe is a giant superposition of waves, representing an unbroken wholeness, of which the human brain is a part (Bohm, 1990). Accordingly, the individual mind or consciousness is an inherent property of all matter (and energy), and as such being part, or rather an expression, of this universal quantum field, the apparently diffuse time/space localization of mental functions argues in favor of an underlying multidimensional space/time reality.

Quantum theory describes the fundamental level of energy and matter, such as mass, charge and spin that are given properties of the universe, not capable of further reduction or explanation. In quantum theories of consciousness, it is suggested that consciousness is such a fundamental property existing at this level. Some theories are additionally linked to the structure of space time, such as the smooth continuous curvature of space that describes gravity in general relativity is incompatible with the discreteness of particles/waves that is fundamental to quantum theory. But it was shown in my article "The Complex Model of The Quantum Universe", IOSR-JM, ISSN: 2278-5728. Volume 4,Issue 1(Nov. - Dec. 2012), PP 20-33, www. iosrjournals.org. the energy densities of the two phases are directly proportional [i.e. $\boldsymbol{\varepsilon}_{\mathbf{1}}(\mathrm{t}) \propto \varepsilon(\mathrm{t})$ ] which indicates that the large or small matter energy density 
in the vapor phase (so called nothing) changes to the large or small matter energy density in the liquid phase (Einstein's 4-dimensional universe) and hence it is found, an important fact, that the existence of discrete structure in the universe, ranging from galaxies to super-clusters. Again classical physics comprises only so-called billiard ball relationships, with bits of matter and energy bumping into one another. These relationships are local, in that they involve immediate contact. Such relationships are also normal in quantum physics. Non locality is the remaining special feature of quantum theory. This applies only where two particles have been in some close relationship, such as two electrons in the same orbital. In very recent experimentally proved that it is possible such as two electrons remain in the same orbital. In this case they can become correlated. For instance the spins of two particles are in a wave form, as both will be in a superposition of up and down. However, if the wave function of one particle collapses, that particle chooses one or the other superposition. When that happens, the other particle will choose the opposite position. It was shown experimentally by Thomas A; "For a basic introduction to QM". This happened when two particles are out of range of a signal travelling at the speed of light. No matter, energy or conventional information is transferred, and the experiment is not regarded as a violation of relativity, but it is demonstrated that quantum properties can correlate instantaneously over any distance.

The concept implies entanglement (non locality) means the apparently diffuse time space localization of mental functions argues in favor of an underlying multidimensional space-time reality. So that the

Potential energy to couple wave information to mental processes, proposes that wave information is transmitted from and into the brain by wave resonance. According to Vattay et al. 2012; Hagan et al. 2002. The quantum system (of the brain) interacts with a quantum environment, the phase information is lost and cannot be reassembled. By entanglement, the quantum coherence in a small region, e.g. the cell or the brain, might have spatial long-range effects.

\section{Consciousness Studies of $\mathbf{2 0}^{\text {th }}$ Century}

The traditional explanation for consciousness or the soul is known as dualism, i.e. a separate spirit stuff and physical stuff, with the spirit stuff capable of acting on the physical stuff, as when the soul commands the body. Again the study of emotions being largely prescribed, with brains conceived as being reasoning machines and nothing else. But the failure of dualism is one of the few points that identify consciousness with a fundamental of the universe (Thompson, 2000). In the 1990s, the dominant explanation for consciousness is driven by the success of computers as problem solving and memory storage machines. The main proposal is that any system or machine that processes information in the same way as the brain will be conscious, regardless of what it is made of. But it does not explain how consciousness arises in the brain and nor does it explain how consciousness might be at some point arise in silicon or other matter. It is very interesting point that carbon is an essential element for the building of live body or human body etc. and it is clear that carbon \& silicon lying in the same group of the periodic table of chemistry. So, as consciousness arises in the brain of human or lives in a biological temperature which was made on the basis of carbon, then it is possible that consciousness may arise at some point if there any creation of lives/anything is made on the basis of silicon within the required temperature. The most popular "Identity Theory" declared that consciousness was identical to the brain or identical to its processing. However, it explain why it was identical to the brain, but not to any of the other physical structures in the universe. Nor did it attempt to define what it meant by the brain, despite the fact that our understanding of the physical processing of the brain was changing dramatically. It was further undermined by the discovery that much neural processing such as the dorsal stream governing spontaneous movement could be brought to completion outside of consciousness, which was seen to be more closely related to longer-term evaluations and planning.

The next popular idea of consciousness was the Epiphenomenalism. The theory proposes that consciousness is a by-product of neural processing that has, however, no function. Which conflicts with evolutionary theory in that it is hard to see why evolution should select for something that had no function, particularly as neural processing is exceptionally energy-hungry. The theory also conflicts head on with physics in which there is no causality, with every object or process having influences elsewhere and finally functionless by-product, there is still no physical evidence for what produces such a thing in the brain. In the present century, it now accepted that mental events could influence the body and that visceral events could feed back on the brain. It also accepted that emotion is a relevant aspect of mental life. However, there was an over-reach in suggesting that the body somehow drove consciousness that the brain could not produce. This seemed to assume some kind of undefined special property in the body that was not present in the 
brain. More specifically it ignored the fact apart from the sense of touch signals entered the brain directly from the environment and were consciously processed in the higher sensory and frontal cortex before being signaled to the viscera. A popular concept of consciousness is an emergent property. Emergence is a familiar process in physics. Liquidity is an emergent property of water. The individual component hydrogen and oxygen atoms do not have the property of liquidity. However, when they are bound together in a sufficiently large number of water molecules, the property of liquidity emerges. The energy particles in the symmetry of SU(11), can be compared with emergence theory make a change after symmetry breaking into $\mathrm{SU}(6), \mathrm{SU}(5), \mathrm{U}(1)$ with dualism, where spirit stuff is due to SU(6) and physical stuff is due to SU(5). The spirit stuff of consciousness (including intelligence) is due to $\mathrm{SU}(6)$ formed mass body with $\mathrm{SU}(5)$ [having the component as strong force $\mathrm{SU}(3)$, weak force $\mathrm{SU}(2)$ ], and electrodynamics $\mathrm{U}(1)$ with fermions. Thus quanta of $\mathrm{SU}(11)$, is divided into two main classes like physical stuff and spirit stuff which possesses mass \& soul in our real/physical universe and then gradually consciousness will be objective with intelligences etc. according to the liquidation of the universe. According to Murphy, 2007, 2011, Auletta et al, Clayton and Davies, 2006, anybody who thinks that consciousness is an emergent property is possible in physics could simplify their search for consciousness by accepting the idea of dualistic spirits. Quanta are unlike any particles or objects that are encountered in the large scale world. When isolated from their environment they are conceived as having the property of waves, but when they are brought into contact with the environment, there is a process of de-coherence, in which the wave function is described as collapsing into a particle. The wave form of the quanta of SU(11) is different from waves in matter of SU(5) in the large scale world, such as the familiar waves in the sea. These involve energy passing through matter. By contrast, the quantum wave can be viewed as a wave of the probability for finding a particle in a specific position. This probability wave also applies to states of the quanta such as momentum. While the quanta remain in its wave form, it is viewed as a superposition of all the possible positions that the particle could occupy. At the peak of the wave, where the amplitude is greatest, there is the highest probability of finding a particle, when the wave eventually collapses. However, the choice of position for each individual particle is completely random, representing an effect without a cause.

\section{Appearance of Our Universe}

There is no consensus yet on how the universe initially came to be, the general assumption is that perhaps an energetic fluctuation caused the universe to tunnel into the existence from quantum foam. The question of why the large energy of the universe is in a dark, i.e. not found in practical, the observed vacuum energy is so small in comparison to the scales of particle physics is known as cosmological constant problem. It is generally thought to be easier to imagine an unknown mechanism which would set vacuum parameter exactly to zero and so it can be considered that there exist several unifications from the very early universe. This class of symmetry group can be expressed mathematically as $\mathrm{SU}(11) \supset \mathrm{SU}(5) \times \mathrm{SU}(6) \times \mathrm{U}(1)$; $\mathrm{SU}(23) \supset \mathrm{SU}(12) \times$ $\mathrm{SU}(11) \times \mathrm{U}(1)$; SU (47) $\supset \mathrm{SU}(24) \times \mathrm{SU}(23) \times \mathrm{U}(1)$; ...........so on. We can assume $\mathrm{SU}(5)$ as matter energy group and $\mathrm{SU}(6)$ a new type of energy sources may be called as latent energy group together which changes into the energy group SU(11) [similarly, apply for SU(23); SU(47) etc.], i.e. to the super unified group. So, it is considered that the breakdown of SUT (Super Unified Theory) symmetry group SU(11), breaks into fundamental group $\mathrm{SU}(5) \times \mathrm{SU}(6)$ leads to a phase transition and then the fundamental group $\mathrm{SU}(5)$ which also breaks into subgroups $\mathrm{SU}(3) \times \mathrm{SU}(2) \times \mathrm{U}(1)$, in which the scalar field $\Phi$ changes. The original vacuum, i.e. false vacuum $(\Phi=\sigma)$ is no longer the true vacuum $(\Phi=0)$. The inflationary stage arises, however, if the true vacuum is not immediately attained. It can explain how the universe appears from nothing. It seems our universe comes from nothing to the universe belongs to the energy group $\mathrm{SU}(11)$, a special unitary group having $11 \times 11$ matrices, such that $\mathrm{SU}(11) \supset \mathrm{SU}(5) \times \mathrm{SU}(6) \times \mathrm{U}(1)$. Thus, we have from $\mathrm{SU}(11)$, the Hermitian matrix $\mathrm{H}$ has 120 arbitrary constants. Which correspond to 120 bosons that now mediate between the different basic entities, of these we already have 60-bosons from the matter energy group SU(5), latent energy group SU(6) and from $\mathrm{U}(1)$. Thus, $120-(24+35+1)=60$ more bosons are namely J-bosons are expected to link the participants of $\mathrm{SU}(6)$ with $\mathrm{SU}(5)$. Therefore, in the theory of $\mathrm{SU}(11)$, it is possible to change any of 30 (thirty) latent energy bosons of SU(6) into any of the 30(thirty) matter energy bosons of $\mathrm{SU}(5)$ or vice-versa by the exchange of the Jboson. That is why it becomes possible to create or destroy matter particles and hence the universe seems to appear from nothing. In the present dissertation there is neither any starting point nor any ending point of the wider (measurable in quantum cosmology) universe (i.e. complex space-time). Only there exists the initial and end conditions for narrower (measurable classically) universe (i.e. Einstein space-time) which emerged from wider universe by the process of changing phase, where it is a continuous process. Again, on the other-hand, the idea that our 4-dimensional universe might have emerged from a higher dimensional space-time is now receiving much attention where the compactification of higher dimensions plays a key role. However, the 
question arises of how and why this compactification occurs. From string theory we know that the compactification may take place provided that the higher dimensional manifold admits special properties, namely if the geometry of the manifold allows, for example, the existence of a suitable killing vector. However, it is difficult to understand why such manifolds are preferred and whether other possible mechanisms for compactification do exist. In cosmology, on the other-hand, different kinds of compactification could be considered. For example, in an approach, called dynamical compactification, the extra-dimensions evolve in time towards very small sizes and the extra dimensional universe reduces to an effective 4-dimensional one. This type of compactification was considered in my previous published paper ["The complex model of the universe" of IOSR-JM, vol.2, 4 (2012), pp-41] with the help of Modern Kaluza-Klein theories. It is then a natural question that how an effective four dimensional universe evolve in time and whether the resulting cosmology is similar to the standard FRW four dimensional without extra dimensions. A universe is based on the considerations of dark energy sector. One may start from a fundamental theory including both gravity and standard model of particle physics. In this regard it is interesting to begin with $10(=4+6)$-dimensional spacetime, in which case one needs a compactification of 10-dimensional super gravity theory where an effective 4dimensional undergoes acceleration. However, it has been known for some-time that it is difficult to derive such a cosmology and has been considered that there is a no-go theorem that excludes such a possibility, if one takes the internal space to be time-independent and compact without boundary. However, it has recently been shown that one may avoid this no-go theorem by giving up the condition of time-independence of the internal space, and a solution of the vacuum Einstein equations with compact hyperbolic internal space has been proposed based on this model. Similar accelerating cosmologies can also obtain for SM 2 and SD 2 branes, not only for hyperbolic but also for flat internal space. On the other-hand, from cosmological point of view it is not so difficult to find cosmological models in which the 4-dimensional universe undergoes an accelerating expansion and the internal space contracts with time, exhibiting the dynamical compactification. In my paper we consider the internal space as imaginary (pseudo-space, $b=i R_{I}$ ), where the matter belongs to another phase by the phase transition process with the help of the latent group $\mathrm{SU}(6)$. It is shown that using a more general metric and introducing matter without specifying its nature, the size of compact space evolves as an inverse power of the radius of the universe. The FRW equations of the standard four dimensional cosmology is obtained using an effective pressures expressed in-terms of the components of the higher dimensional energy-momentum tensor and the negative value of this pressure may explain the acceleration of our present universe. One of the recent important cosmological observations is the accelerated expansion of the universe. It is known from the recent distance measurement of type Ia supernova suggest strongly an accelerating universe. This accelerating expansion is generally believed to be driven by an energy source called dark energy which provides negative pressure, such as a positive cosmological constant, or a slowly evolving real scalar field called quintessence. Moreover, the basic conclusion from all previous observations that 70 percent of the energy density of the universe is in a dark energy sector, has been confirmed after the recent WMAP. That means the matter content of the universe is composed of 70 percent of an un-clustered component of negative pressure (dark energy) and 30 percent of a clustered component of zero pressure (cold dark matter). The traditional candidate of dark energy are the cosmological constant, $\Lambda$ and quintessence. But, these approaches have some serious drawbacks from fundamental point of view. In fact, the observed energy density of the cosmological constant has a discrepancy of about 120 orders of magnitude with the theoretically predicted value from field theory models. In the present dissertation, we consider in the very early universe up-to the Big-Rip singularity, there were several unifications. This class of symmetry group can be expressed mathematically as follows: SU (11) つ SU (6) $\times$ SU $(5) \times \mathrm{U}(1) ; \mathrm{SU}(23) \supset \mathrm{SU}(12) \times \mathrm{SU}(11) \times \mathrm{U}(1) ; \mathrm{SU}(47) \supset \mathrm{SU}(24) \times \mathrm{SU}(23) \times \mathrm{U}(1) ; \ldots \ldots \ldots . . . .50$ on. Thus we

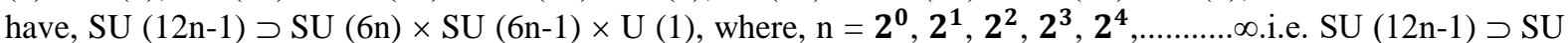
$(6 \mathrm{n}) \times \ldots \ldots \ldots \ldots . . . . . . \mathrm{SU}(24) \times \mathrm{SU}(12) \times \mathrm{SU}(6) \times \mathrm{SU}(3) \times \mathrm{SU}(2) \times \mathrm{U}(1)$. Hence, according to the law of symmetry breaking of the energy group, i.e. according to the symmetry breaking of the Gaussian energy group, there exists neither any Big-Bang nor any Big-Crunch singularity, only there Big-Rip singularity and Big-Break singularity. All the dark energies SU(12), SU(24), ...... etc. Are also responsible for the various consciousness and intelligence of the living cells or bodies according to the progress for biological revolution as such the progress of the material universe depends on the sub-energy groups of $S U(5) \supset S U(3) \times S U(2) \times U(1)$ stated by the standard model of physics. Again the activities of the energy groups for consciousness are restricted to unfold for each galaxy of the universe as well as for every solar system of the galaxies. Our physical universe, starts from SU(11), classically from 10-dimensional space-time, the super-symmetry of the universe. The quantum wave energy universe actually starts from Big-Rip singularity it was shown there exists energy pressure and densities simultaneously. Most probably, $28 \%$ or $30 \%$ of the total energy (measuring by W-MAP) were found for the symmetry breaking of the energy group SU(11) and rest $72 \%$ or $70 \%$ of the energy belongs to another phase (according to the classical mathematics which are folded) starting from Big-Rip singularity due to symmetry breaking of $\mathrm{SU}(23), \mathrm{SU}(47), \ldots \ldots \ldots . .$. 


\section{Double Slit Experiments}

The physicist Richard Feynman said that this classic experiment contained all the problems of quantum theory. In the early nineteenth century, an experiment by Thomas Young showed that when a light source shone through two slits in a screen, and then onto a further screen, then a pattern of light and dark bands appeared on a further screen, indicating that the light was in some places intensified and in other reduced or eliminated. Later, the experiment was refined. Now be performed with one or two slits open. If there was only one slit open, the photons or light quanta, or any other quanta used in the experiment behaved like particles. They passed through the one open slit, interacted with the screen beyond and left an accumulation of marks on that screen, signifying a shower of particles rather than a wave. But once the second slit was opened, the traditional interference pattern, indicating interaction between two waves, reappeared on the screen. The ability to generate the behavior of either particles or waves, simply according to how the experiment was set up, showed that the quanta had a perplexing wave/particle duality. What is remarkable is that with two slits open, but the photons impacting one at a time, the pattern on the screen formed itself into the light and dark bands of an interference pattern. Somehow the photons 'knew' to arrange themselves into a pattern indicative on the interaction of waves. The question arose as to how the photons emitted later in time 'knew' how to arrange themselves relative to the earlier photons in such a way that there was a pattern of light and dark bands, indicative of interacting waves. For the solution of the above problem, we need a new concept about quantum theory, as well as locality and non-locality which hinges by the EPR (Einstein, Podolsky, and Rosen) experiment. Locality comprises the idea of normal cause and the effect under which objects or particles move or change as a result of being impacted by other objects or particles, or of being directly acted on by energetic forces such as the electromagnetic force. The non-locality involves the ability of one particle to determine the behavior of another distant particle instantaneously, and without any matter or energy passing between the two. Einstein termed this 'spooky action at a distance'.

It may be explained by a series of new energy sources $\mathrm{SU}(6), \mathrm{SU}(12)$, ....etc. which may be established the concepts of reality or objective existence of the above mentioned experimental theorem. Some of the new energy sources exist which are not being observed by an observer practically. Our 4-dimensional matter/particle universe or physical universe observed when there was created a "whole", like single slit open i.e. by the symmetry breaking of the unified group SU(5) and creates a shower of photons \& quanta of the matter particles on the natural screen of the universe or otherwise like double slits open from the 10-dimensional space-times photons \& quanta by the new energy sources of SU(6) emitted as 'knew' [i.e. the symmetry breaking of the superunified energy group $S U(11)$ and then $S U(5)$ ], we found an entangled-like matter energy with spirit energy. It was compared with a situation of the universe where relatively very lower energy density in the ocean of the new energy sources in one phase, starting from Big-Rip singularity like as in the gaseous phase and then vapor \& liquid phase. Again the Copenhagen Interpretation of Neils Bohr denies that sort of reality to the wave form of the quanta. It is observed from the Einstein's spacetime theory, that means we have from the Friedmann equation for the evolution of the cosmic scale factor $\mathrm{R}(\mathrm{t})$ which represents the size of the universe, is found at a finite time in the past $\mathrm{R}$ must have been equal to zero. Then the contents of all the galaxies must have once been squeezed together in a small volume i.e. numerically, the volume of the matter universe once been squeezed in a zero volume. We may assume that the matter universe is then transferred into another phase by the phase transition system with the help of latent energy group $\mathrm{SU}(6)[\mathrm{SU}(11) \supset \mathrm{SU}(5) \times \mathrm{SU}(6) \times \mathrm{U}(1)]$. The subgroup $\mathrm{SU}(6)$ has been interpreted as a new type of energy sources other than $\operatorname{SU}(5)[S U(5) \supset \operatorname{SU}(3) \times \operatorname{SU}(2) \times U(1)]$, the standard model of physics. So there were several environmental atmosphere according to the unified group $\mathrm{SU}(11), \mathrm{SU}(23) \ldots \infty$ etc. all belonged to so called "nothing" as said by Einstein. By the last environmental situation produces quanta by the symmetry breaking of the unified energy group SU(5) and formed our physical universe. Each and every environmental situation is formed by the different quanta with the energy sources SU(6), SU(12),...etc. and effect the next generated quanta within their individual environment by the process like wave function collapses. For example we compared the symmetry groups SU(5), SU(11), SU(23),...etc . like with liquid, vapour, and gases states of matter. We assumed that our universe appeared from something instead of nothing. It was discussed in my article 'The Complex Quantum and Classical Pseudo-Tachyonic Universe' [IOSR Journal of Mathematics (IOSR-JM) e-ISSN: 2278-5728, p-ISSN: 2319-765X, Volume 8, Issue 3(Sep. -Oct. 2013), PP 15-32] that the quantum behaviour having some classical counterpart that can always choose such a quantum state, which does not disappear at any value of $\mathbf{R}_{\mathbf{I}}$, which guarantees the positivity of the quantum average of the operator. Coming back to our cosmological model we can say that the 
requirements of the well-definiteness of the pseudo-tachyonic part of the Hamiltonian operator in the Wheeler-DeWitt equation, does not imply the disappearance of the wave function of the universe at some values of the variables and thus, does not reveal the effect of the quantum avoidance of the cosmological singularity.

The strength of weak force gradually decreases and strength of strong force increases as we advanced from the symmetry breaking of the unified group SU(5). The first stage of the formation of our universe from so called nothing which was basically from non-locality to locality by the symmetry breaking of the SUT group SU(11), where the energy group SU(6) remains latent and behaves like as consciousness relative to the energy group SU(5). The bosons of the energy group SU(6) always controlled all measurable energies of SU(5) including 'quarks' \& 'leptons' etc., which comes from pure energy stays in the form like vapor (i.e. in another phase) and hence from the symmetry breaking of SU(11) with the energy group SU(12), we found new types of quanta with conscious energy, quark-like, \& lepton-like particles etc. and specially produces a strong neutral current which are applicable within 'live' cells/elements/animals/plants etc. with an electromagnetic interaction created by $\mathrm{SU}(6)$ with $\mathrm{U}(1)$ in the frame-work of $\mathrm{SU}(6) \times \mathrm{U}(1)$ etc. called spirit stuff, and then from SU(5) are found matter energies, fermions, charge particles, spin, etc. like the decomposes of water by applying electric charges (extra energy) connecting with an electric circuit, we get hydrogen, oxygen as charged particles primarily and then gases with different characters from the characters of water. But the two gases may again react with each other at some situation and get water having no characters either of Hydrogen or Oxygen. It is very interesting that the generated hydrogen and oxygen from water can be reacted with other chemical elements in the some situations and found different characters in different forms. So, we consider, there exists two phases of the universe, (1) local, that means, particle produces as per requirement of the second phase i.e. as per the requirements of our universe which maintain the law of wave-particle duality theorem (physical stuff) and (2) non local, i.e. particles in the wave form (called spirit), produces in the first phase of our universe which controlled the second phase, our physical universe.

All the symmetry breakings from infinite space-time [i.e. Big-Rip singularity] to SU(5) belongs to the phase (like gaseous \& vapors), pseudo-tachyonic \& tachyonic universe. Hence all the energy groups from infinites dominantly effects ourselves while the energy group SU(6) directly controls the whole universe including ourselves creating as consciousness or intelligence and creating others, like emotions etc. and with the collaboration of atoms formed by the energy group of SU(5) produces biological particles. So, by the collapses of quanta with the strong forces of SU(6) within the environmental situations of SU(11) where 30-bosons of SU(6) changes to the 30bosons of SU(5) or vice-versa, unfolded the physical universe and then we found everything of our universe. It is a continuous process from infinitely discrete universe. In recent decades, de-coherence has become particularly popular as a substitute instead of the traditional 'measurement' which always referred to in the Copenhagen version. In de-coherence, the collapse of the wave function happens of its own accord, as a result of the wave becoming entangled with the rest of the environment. In some recent versions, it is suggested that there is no collapse, the information in the wave simply gets lost in the larger scale environment and a branching of reality into separate universes. So in the Schrodinger cat paradox, for instance, the universe splits into one universe with a live cat and one with a dead cat.

The success of the above new quantum explanation emphasized three problematic solutions of quantum theory, a causality in the randomness of the wave function collapse, a causality in the non-local influences demonstrated by EPR (Einstein, Podolsky, Rosen) type experiments and the resulting of agreement as to the underlying reality of the physical universe. So, at the quantum level, we find the sufficient cause of formation the properties of mass, charge and spin that are given properties of the universe.

Again, we consider a (4+D)-dimensional Friedmann-Robertson-Walker type universe having complex scale factor $\mathrm{R}+\mathrm{i} \mathbf{R}_{\mathbf{I}}$ where $\mathrm{R}$ is the scale factor corresponding to the usual 4-dimensional Universe while $\mathbf{R}_{\mathbf{I}}$ is that of D-dimensional space. It is then compared with $(4+\mathrm{D})$-dimensional Kaluza-Klein Cosmology having two scale factors $\mathbf{R}$ and $\mathrm{a}\left(=\mathbf{i} \mathbf{R}_{\mathbf{I}}\right)$. It is shown that the rate of compactification of higher dimension depends on extra dimension ' $\mathrm{D}$ '. The Wheeler-DeWitt equation is constructed and general solution is obtained. It is found that for $\mathrm{D}=6$ (i.e. in 10 dimension), the Wheeler-DeWitt equation is symmetric under the exchange $\mathbf{R}_{\mathbf{I}} \leftrightarrow \mathrm{R}$. Which explains the most popular M-theory/string theory, Viewing particles and space-time as dual aspects of the same thing [See IOSR Journal of Mathematics ISSN: 2278-5728 Volume 2, Issue 4(SepOct2012), PP 41 www.iosrjournals.org "The Complex Model of The Universe"].

Hence the quanta and related space-time appeared to the physical universe where it might be possible for science to insert consciousness is an additional fundamental property, (explained in the reviews Vannini and Di Corpo, 2008 Hu and Wu, 2010, and Tarlaci, 2010,Meijer and Korf, 2013, Pereira, 2003 Atmanspacher, 2011). 
Again, Georgiev, 2003 discusses epiphenomenalism, the theory that consciousness is a by-product of brain processing having only an illusion of causal influence. He points out the evolutionary argument against this view, to the effect that evolution would not select for something that conveyed no selective advantage. He sees the idea that we have no freedom or moral responsibility as counterintuitive. Such a counterintuitive result is seen as the inevitable consequence of explanations based on deterministic classical physics. Quantum mechanics does, however, provide a non-deterministic alternative, in which consciousness underlies the neural processes of making choices and thus effecting future possibilities. Max Tegmark asserted what was already an established position to the effect that quantum coherence in the brain would be too short lived to have a functional role in neural processing (Tegmark, 2000). Although an orthodoxy, to the effect that quantum coherence cannot occur in organic matter.

Tegmark's paper was aimed at refuting Hameroff's Orch OR theory, (Hameroff and Penrose, 2011) which required quantum coherence to be sustained for $25 \mathrm{~ms}$. Thus Tegmark did not show that coherence over shorter timescales could not support consciousness, because he was directing his argument at the longer timescales of Hameroff's theory. Again Tests shows that there is a minimum timescale of about $30 \mathrm{~ms}$ needed for a subject to distinguish two sensory inputs as being separate. This means that consciousness cannot be slower than 30ms. However, patients with time agonise, who have subjective experience of the passage of time, confirm that it is physically possible to have consecutive conscious steps that are experienced as simultaneous. From this it is argued that the real units of consciousness could be at the picoseconds level, although such units cannot be discerned by the conscious subject. So, the upper possible bound of the timescale of consciousness need not be its actual scale. As an analogy, Georgiev takes computer screen is on a millisecond timescale with the screen refreshing perhaps every 10ms. But this is not indicative of the performance of the processor, which may 18 operate on a picoseconds timescale. The refresh rate of the monitor can tell us that the processor does not operate on a slower than $10 \mathrm{~ms}$ timescale. In the brain, the millisecond timescale applies to the brain's communications with sensory organs and muscles, but this may not say much about its internal processing.

\section{Quantum Neurobiology}

We have from the discussion of Meijer and Korf, 2013, the idea that the physical quantum concept do physically apply to the mind: the mental domain is considered as an aspect of wave information and a special position takes the feature of "superposition": quantum particles can be present in the multiple spatial locations or states and be described by one or more pure state wave functions simultaneously of which a single state can finally be selected. Although Penrose, (1989) suggested that the underlying space /time geometry in fact bifurcates during the superposition process and wave collapse occurs in a non-computable manner. It was suggested the conditions found in the microtubule could allow coherent quantum particles to form a unity that can be described by a single wave function. These concepts are considered 'the hard quantum theories', as opposed to the "soft" or formal theories. If someone takes the quantum model of the human mind serious and even if someone is convinced by the admittedly weak empirical evidence for retro-causality during unconscious processing, still there are some quite good theoretical arguments that speak against the existence of retro-causal effects. One is related to the time travel paradox that is involved in retro-causal information transmission and another one is related to the impossibility of supraluminal signal transfer postulated by special relativity. The eminent quantum scientist who suggested that the mental and the material domain are governed by common ordering principles, and should be understood as "complementary aspects of the same reality"(see Atmansapacher and Primas 1977; Primas 2003). The "hard" mental QM theories apply either to specific brain structures/molecules (this section) or to quantum fields and dimensions or both. Vannini and Di Corpo distinguish models based on consciousness creating reality, models based on probability aspects of QM and models based on already established QM order principles. Hu and Wu differentiate in models based on QM elements of entanglement and coherence and models on the relation of QM with consciousness that can include materialistic modes (consciousness emerges from material brain), dualistic mind/matter models and panpsychistic modes. More detailed reviews can be found in Pereira, 2003 and Tuszynsky and Woolf, 2010, the latter as an introductory chapter of the instructive book: "Emerging Physics of Consciousness", while an excellent and critical overview of the field is provided by Atmanspacher, 2011. It should also be kept in mind that classical physics can be fully derived from quantum physics. I think which actually started from infinite space-time called Big-Rip singularity [see IOSR Journal of Mathematics (IOSR-JM) e-ISSN: 2278-5728, p-ISSN: 2319-765X, Volume 8, Issue 3(Sep.-Oct. 2013), PP 15-32 www.iosrjournals 'The Complex Quantum and Classical Pseudo-Tachyonic Universe'], where the energy density \& pressure exists. The present situation of the universe emerging from last symmetry breakings of the energy groups SU(11) and then SU(5) through a long continuous process from the discrete structures of the whole universe. We found so many interpretations, as Copenhagen, Many worlds, Implicate order, Interact ional theory, Micro-macro-scale definition, Environmental de-coherence, Relational quantum mechanics etc. Yet a number of common elements 
such as the true particle/wave aspect, instead of only a probability function, (Pusey et al., 2012), superposition, entanglement/non-locality and coherence/de-coherence phenomena are experimentally established and remain very usable in practice. It is often stated that quantum wave information coherence cannot be maintained long enough in the brain due to interaction with the macro-environment of the brain components. Yet, on this point major differences in de-coherence-time calculations exist, as based on various models and their intrinsic assumptions (see Hagan et al., 2002 and Tegmark, 2000, Lloyd, 2011). A central point here is that subcompartments could be present at the molecular or sub-molecular level, that by their special arrangements of quantum noise protected or coherence stabilized. Examples are internal parts of channel proteins (Bernroider, 2004), and stabilization by clustered (gel/sol) arrangements of cytoplasmic water clusters (see Hameroff and Penrose, 1996, Penrose and Hameroff, 2011). Through coherence and macroscopic entanglement, life time of wave information can be much longer than in the classical phase, as a consequence of coherence/de-coherence dynamic equilibrium, allowing non-local remote interaction in large number soft entangled neurons. Such gel/sol oscillations could even be a primary to excitation/depolarization triggered by normal sensory stimuli and are supposed to interact with zero-point vacuum dipole vibrations (the bi-vacuum matrix model of Kaivairanen, 2006).

It was explained that the empty/vacuum space, is not absolutely empty/vacuum, it was filled with some unexplained new energy quanta of quark-like \& lepton-like particles with strong new energy sources of Gaussian group $\mathrm{SU}(6), \mathrm{SU}(12), \ldots .$. etc. which controlled the creation and behaviour of quark \& lepton with charge particles and hence electro-magnetic force [see 'The Complex Quantum and Classical Pseudo Tachyonic Universe']. It should be realized that de-coherence, does not, per definition imply destruction of information since, firstly, it is not compatible with the quantum principles of non-cloning and non-deletion, secondly a cyclic process of de-coherence and re-coherence processes cannot be excluded (see Hartmann, et al 2006; Li and Paraoano (2009);Atmanspacher, 2011) while thirdly, even if such de-coherence does occur, it may result in mixture of possibilities that may be accommodated by the collection of perceivable worlds in the brain (Stapp, 2012). It has been proposed by Vattay and Kauffman, 2012, that a de-coherent state can be converted back to a coherent state by the input of adequate phase and amplitude information. The resulting coherent states can last long enough in warm biological systems in order to, for example, enable coherent search processes for antennamediated transport of photon energy in photosynthesis. As there also exists photon-like particles of SU(6), so in the micro domains, on the edge of chaos, could also be instrumental in the human brain as sites where a dynamic interplay of de-coherence and re-coherence takes place. Evidence was more recently presented that quantum physics can be applied to macromolecules (Zeilinger, 2000), and to the surprise of many, even can occur in warm and wet biological systems (photosynthesis: Engel et al., 2010) and brains of birds in relation to magnetic sensing and navigation (for references see Arndt et al., 2009, Lloyd, 2011). Lloyd concluded: "Quantum coherence plays a strong role in photosynthetic energy transport, and may also play a role in the avian compass and sense of smell. In retrospect, it should come as no surprise that quantum coherence enters into biology. Biological processes are based on chemistry, and chemistry is based on quantum mechanics. If an organism can attain an advantage in reproduction, however slight, by putting quantum coherence to use, then over time natural selection has a chance to engineer the necessary biochemical mechanisms to exploit that coherence. Different types of quantum processes that operate at the same time scale can interact strongly either to assist or to impede one another. In photosynthetic energy transfer, the convergence of quantum time scales gives rise to more efficient and robust transport. Evolved biological systems exhibit the quantum Goldilocks effect: natural selection pushes together time scales to allow quantum processes to help each other out. According to Tuszinsky and Woolf, 2010, Meijer, 2014, a spectrum of atoms/molecules has been suggested to operate in a quantum manner: $\mathrm{Ca}^{2+}$ ions and $\mathrm{K}^{+}$ions, $\mathbf{H}_{2} \mathrm{O}$, enzymes, membrane receptor and channel proteins, membrane lipids, neurotransmitter molecules, in addition to macromolecular structures such as DNA/RNA, gap junctions, pre-synaptic vesicles, microtubules and micro-filaments.

Since our integral universe can be described by the current laws of QM and relativity, it should be required a generalized quantum theory starting from infinite space-time and then further of symmetry breaking [see IOSR Journal of Mathematics (IOSR-JM)e-ISSN: 2278-5728, p-ISSN: 2319- 765X.Volume 10, Issue 5 Ver. III (Sep-Oct. 2014), PP 53-68, www.iosrjournals.org 'The Origin of Consciousness in the universe-IOSR]. We get the latest situations of quantum mechanics and hence, it was warranted to place the human brain outside the nature. Even some cosmic architecture mirrored in our complex brain (Kak, 2009; Amoroso, 2003). The discussion around higher brain functions is frequently obscured by modalities of promissory materialism: "at present we do not understand how consciousness arises in our brain" but, we try to realize gradually that the extra-dimensions as explained in my articles are associated with consciousness and now, it is required to identify the characters and behaviours of the bosons of SU(6), ....etc. and the quanta of the first phase, producing with the symmetry breaking of the Super Unified Group SU(11), etc., then we can identify the characteristics of the consciousness. It may be considered that any type of expressions [as like emotions, sentiments, angry, etc. for humans] of humans, animals, plants 
etc. is due to some kind of electromagnetic interactions. It is not our purpose to assess the various QM theories in detail rather we intend to discuss some of their major implications regarding the concept of a "quantum brain". Several QM theories are based on specific properties of proteins, as for instance micro-tubular proteins, (Penrose, 1989; Hameroff, 2007), proteins involved in facilitating synaptic transmission (e.g. Beck and Eccles 1992; Beck 2001), including $\mathbf{C a}^{+2}$-channels, see Stapp, 2009), as well as specific channel proteins, instrumental in the initiation and propagation of action potentials ( $\mathbf{K}^{+}$channels), Bernroider and Roy, 2004), QM theories also extends the mind to different spaces and time dimensions and some consider the individual mind (partly) as an expression of a universal mind through holonomic communication with quantum fields. The human brain is conceived as an interfacing organ that not only produces mind and consciousness but also receives information necessary for full deployment of these mental phenomena. The particular local collapse of the wave function, in this manner, produces new information. As originally proposed by Eccles, this is realized by membrane protein induced fluxes of $\mathbf{C a}^{2+}$ or $\mathbf{K}^{+}$ions, that than increase the probability of fusion of neurotransmitter-filled vesicles in the synapses, leading to the firing of the particular neuron or even groups of neurons. The central hypothesis here is that synaptic transmission represents a typical (quantum) probability state in which the total number of vesicles available for exocytosis is critical for an all or none response of neuronal firing (Beck and Eccles, 1992; Beck, 2001). Coherent neuronal perturbations and especially their entangled state are supposed to provide nonlocal "binding" of sensory and cognitive brain centres, and may also enable perception of qualia and the unitary sense of "conscious self" (Hameroff, 2007). As the "mesoscopic" scale of brain activity where the "binding" process is expected to occur is in the vicinity of the quantum domain, the binding principle is likely to be a quantum non-local effect, probably the only known physical mechanism able of performing such a task. One possibility is the formation of a quantum photonic field (Flanagan, 2006); another possibility is the formation of coherent states on the level of trans-membrane ion fluxes such as that of $\mathbf{C a}^{2+}$ ions as suggested by Pereira, 2003, 2007. The model of Davia, 2010 (see the chapter in the book edited by Tuszinski and Woolf, 2010), proposing that consciousness is related to travelling waves in the brain as a uniting life principle on multiple scales, which is based on energy dissipation, enzyme catalysis, protein folding that maintains energy balance in an excitable system such as the brain, conditions that are also compatible with the iso-energetic brain model treated in Meijer and Korf, 2013. Penrose, 1989; Hameroff, 2007 have discussed that microtubule, in principle, can maintain quantum states (i.e. superposition) lasting at least $\mathbf{1 0}^{-\mathbf{6}}$ seconds, long enough to be instrumental in the transfer of quantum wave information. Such lasting quantum states are possible because of the shielding of hydrophobic pockets in the particular proteins, as well as the formation of coherent clusters of these molecules that thereby share a common quantum wave function (so called Bose-Einstein Condensates). There is indirect evidence that microtubules may be relevant for neuro-cognition: increased synthesis in relation to postnatal development with regard to synaptogenesis and visual learning and as counterpart aging deficits in memory as well as interactions with general anaesthetics (Penrose and Hameroff, 2011, 2012; Tuszynski and Woolf, 2010; Kalvairainen, 2005). Yet such correlative studies should not only be further substantiated with experiments that show quantum states in isolated tubules, as reported by (Bandyopadhyay, 2011), but rather and most importantly, directly demonstrate tubular involvement in higher brain functions. .More recently in 2013 Bandyopadhyay's group demonstrated that in microtubules the energy level of up to 40,000 individual tubulin proteins and the energy level of the microtubule are the same. The water core and the individual tubulin proteins are suggested to control the properties of the microtubule by means of delocalised electromagnetic oscillations. The properties of the microtubule might be taken to suggest that the system can support a macroscopic quantum state.

In conclusion: tubular and synaptic channel proteins exhibit conformational transitions within $\mathbf{1 0}^{\mathbf{- 9}}$ seconds that may last for $\mathbf{1 0}^{\mathbf{- 6}}$ seconds or even longer, (Beck and Eccles 1992; Beck 2001; Bernroider and Roy, 2004, Kaivairainen, 2005). These perturbations may last long enough to be finally detected as miniature neuronal potentials (Hamill et al., 1981, Hagan et al., 2002). The particular mechanisms also imply a manifestation of non-local quantum effects due to distant coherence, which was actually produced by the new energy or quanta as expressed in my articles. Again phenomenon that was even recorded in laser stimulated neuronal cell cultures in which classical physical explanations were excluded (Pizzi et al., 2004).

\section{How Consciousness Arises From Quantum-Gravity}

The coherence of such quantum states among brain proteins has been suggested to lead to material changes in brain physiology through orchestrated collapse of quantum coherent clusters of tubulin proteins, triggered by quantum gravity expressed at the spin (Planck scale) level. On the basis of a recent theory on the nature of gravity (Verlinde, 2011), postulating that gravity is not a force but rather an entropic compensation for the movement of mass/information, it was speculated that consciousness may arise from a gravity - mediated reaction on the entropic displacement of information as it occurs in high density in the human brain (Meijer, 2012). Anyhow, there should be a mechanism to integrate signal processing within a single neuron with other, even distant, neurons and consequently non-local effects due to quantum entanglement formed by the 
condensation of the quanta-like from another phase like the phase transition system where the bosons of the group SU(6) controlling the whole systems. Again these quantum processes may explain phenomena such as qualia, meaning, sensation of unity, intentionality as well as conflict solving, reliability in the sense of correspondence with the outer world and the sense of self. The latter is related to the feeling of causal power that could result from a quantum/classical interface in which classical synaptic processes create a quantum coherent state that enables quantum computation that exert a back-influence on the original synaptic process (Pereira, 2003, 2007).

The existence of non-locality in brain function, being a basic property of the universe strongly argues for an underlying deep reality out of space/time as originally proposed by Bohm (1990) in the form of an implicate order. But we may assume that it is associated with the extra-dimensional space-times other than 4dimensional space-times. Bohm claimed that these mechanisms also play a role in different forms of transpersonal and extrasensory perception by wave resonance with universal quantum field (Kak, 2009, Jahn and Dunne, 2007, Kafatos and Draganescu, 2000, Kafatos, 2009). The wave information could be transmitted from and into the brain by wave resonance and may locally collapse to matter entities through conscious observation, including sufficient individual attention and intention (Stapp, 2009).

Stapp (2012) argued recently that this does not represent an interference effect between superposed states, as assumed by Hameroff and Penrose (1996), but that through environmental de-coherence, superpositions will be converted to multiple mixtures of information. Since our brain contains a large collection of perceivable worlds, it is able by super causal free choice and subsequent common random choice to make a fit with one or more of the above mentioned mixed information modalities. The particular waves than spread out and rapid sequence repetitions (the so called Zeno effect) may sufficiently maintain coherence in parts of the brain. Of note, Stapp does not see free will as based on quantum probability aspects. He states: "In the original Copenhagen formulation this extra process is initiated by what is called "A free choice on the part of the experimenter." The phrase "free choice" emphasizes the fact that, while a definite particular choice is needed, this choice is not determined by any known law or rule: The purely physical aspects of the theory have, therefore a significant causal gap, which opens the door to a possible causal input from the mentally side of reality". The existence of a quantum superposition is a linear function of the extent to which information is available about the alternative states that constitute the superposition (Wang, Zou and Mandel, 1991). The more distorted the information about the single states is, and therefore the less classical (0/1) it is, the more ideal a superposition will be.

Quantum information may exert physical effects via a bottom-up flow of information starting at spin networks (Penrose, 1994; $\mathrm{Hu}$ and $\mathrm{Wu}, 2010$ ), that can be passed on as wave forms of elementary particles/atoms, to be ultimately expressed at the level of neuronal molecules. Meijer and Korf, 2013, consider the latter flow of information more feasible than being directly transferred through vibratory interference at the molecular level. According to this integral quantum model, perturbations at the various spatiotemporal domains allow both time-symmetric forward and backward causation and therefore top-down influence of quantum fields. The basic question is: how are quantum waves or quantum fields finally perceived by the human brain and how they influence or even induce phenomena as (self) consciousness? Organisms do indeed visually perceive photons that exhibit wave/particle duality; humans even senseless than ten photons, whereas insects may even detect a single photon (Baylor et al., 1979; Menini et al., 1995). Sensitive detection is possible with dedicated cellular structures as for instance in the mammalian retina that amplify the energy of a single photon by a cascade of processes, based on changes of protein conformations and cellular potential energy, leading to the electrochemical stimulation of neurons projecting to the brain. Recently, photosensitive proteins have been coupled to ion-channel proteins with biotechnical techniques, so that the neural activity can be modified or inhibited in vivo by light introduced via optic micro-fibres (Lima and Miesenböck, 2005; Boyden et al., 2005; Tsai et al., 2009). These experimental approaches demonstrate that quantum effects may directly affect neural function, but it remains to be shown more definitely, that this also does occur directly inside the human brain as it was demonstrated in the brain of birds (see the reviews of Arndt et al., 2009, Lloyd, 2011). It was proposed by Martin (Martin et al, 2013) that archetypes can be stored as quantum systems and that consciousness may be controlled by quantum entanglement from outside of the 4-dimensional space-time. That means higherdimensional space-times.

Our physical universe actually starts the journey from the stages of 10-dimensional space-time. So appears our present 4-dimensional physical universe. All the molecules/atoms biological elements and all physical laws including theoretical \& experimental are explained on the basis of standard model of physics within 4-dimensional space-time. We assumed that, the actual universe were multi-dimensional. All the quanta with photon-like of $\mathrm{SU}(6), \mathrm{SU}(12), \ldots$, constitutes a package of new energy particles or conscious particles which controlled the whole universe with an appropriate consciousness/intelligences as well as created self consciousness within our complete live body. Interestingly, recently a 5-dimensional space-time brane model was proposed in order to adequately position of consciousness and universal consciousness in the cosmos 
(Carter, 2014a, 2014b), an item that was discussed earlier by Smythies, 2003 suggesting that consciousness may be in a brane rather than in the brain. Atmanspacher (2003) explained that mind/matter correlations may require new science, in the sense that the use of emergence and reductionistic schemes may not be adequate and should be replaced by possible symmetry breaking within a domain in which matter and mind are un-separated. $\mathrm{He}$ cites d'Espanat postulating an independent the 'Ultimate Reality' that is neither mental nor material.

According to the Gaussian transformation theory our created universe actually started from the symmetry breaking of the Gaussian group $\mathrm{SU}(11)[\supset \mathrm{SU}(6) \times \mathrm{SU}(5) \times \mathrm{U}(1)]$, from which we get two fundamentally energy groups, $\mathrm{SU}(6)$ and $\mathrm{SU}(5)$, where $\mathrm{SU}(6)$ leads to a phase transition action and then fundamental matter energy group $\mathrm{SU}(5)$ breaks into subgroups $\mathrm{SU}(3) \times \mathrm{SU}(2)_{\mathrm{L}} \times \mathrm{U}(1)$, in which the scalar field $\Phi$ changes. The original vacuum, i.e. with false vacuum $(\phi=0)$ is no longer the true vacuum $(\phi=\sigma)$. The inflationary stage arises, however, if the true vacuum is not immediately attained [see the details explanation in my article 'The complex model of the universe']. SU(6) creates as intelligence/mind of our universe as well as self consciousness with respect to the energy group SU(5), and hence SU(6) controlled the whole universe as quantum mind in the domain of SU(11). Therefore, in the theory of SU(11), it is possible to change any of 30(thirty) photonic bosons of intelligence/ quantum mind of SU(6) into any of 30(thirty) material energy photonic bosons of SU(5) or vice-versa by the exchange of the J-bosons of SU(11) within the same symmetry or domain. That is why it becomes possible to create or destroy material substances and hence matter \& mind are not separated from each other within the same domain of SU(11). Which also explained by Atmanspacher (2003) that mind \& matter are correlated.

So, the famous postulates of d'Espanat: there exists an independent "Ultimate Reality", i.e. neither mental nor material. Although, which explained details in my article "The Origin of Consciousness in the universe- IOSR", that our universe appears by the several symmetry breaking of the energy group from Big-Rip singularity. Now, consciousness should be a property of all living structures in which each biological process is forced to choose between information coming from the past and information coming from the future (King, 2003). We consider such models (including that of Vannini and Di Corpo, 2008) attribute consciousness to principles of generalised relativity theory of higher dimensions $(4+D)$ with complex space-time $\left(R+i \mathbf{R}_{\mathbf{I}}\right)$ and quantum physics with new energy sources of the energy group SU(6). Processing an amplification of quanta/wave information in the brain may underlie the presumed higher brain or mental functions. If one assumes that such detection mechanisms does indeed operate in the brain, then the next question is whether the information to be processed is exclusively associated with quantum waves or quantum states or, alternatively, with the specific proteins that carry them. Apart from discussing the inherent mechanisms such as forward and backward causation, superposition and entanglement in the mental space, we shortly treat the idea that individual mind may, at least partly, be an expression of universal consciousness as opposed to the concept the mind is merely an attribute of matter. It was shown in my articles that the Wheeler-DeWitt equations valid for tachyonic universe as well as pseudo-tachyonic universe, when space-time is $\mathbf{R}+\mathbf{i} \mathbf{R}_{\mathbf{I}}$. That means the Hamiltonian is well defined in the pseudo-tacyonic field in respect to the pseudo-space $\mathbf{R}_{\mathbf{I}}$. Hence, we consider that, if one can always choose such a quantum state, which does not disappear at any value of $\mathbf{R}_{\mathbf{I}}$ which guarantees the positivistic of the quantum average of the operator.

\section{Explanation of Undivided Wholeness of The Universe}

In Bohm's scheme there is an unbroken wholeness at the fundamental level of the universe, in which consciousness is not separated from matter. Bohm says that in the implicate order intermingled elements are present together, and processes are the outcome of what is enfolded in the implicate order. In this structure, there is a flow between experiences and logical thought that is considered by Bohm to hold out the possibility of a bridge between matter and consciousness. Bohm also advances the idea of overall necessity driving short-term brain processes. Thus it is proposed that an ensemble of elements enfolded in the brain will constitute the next development of thought, and that these elements are bound by an overall necessity that brings them together and also determines the next moment in consciousness. Basil Hiley was the long-term associate of David Bohm, (Bohm and Hiley, 1987, 1993). They argue that the Bohmian notion of active information introduced in relation to quantum phenomena can also be applied to classical signalling. This is suggested to have relevance to concept of meaning as opposed to mere information. Hiley queries whether the word 'information' that is widely used in science including neuroscience always carries the same meaning. Bohm and Hiley were interested in so-called active information that drives physical processes and leaves no choice as to whether they are implemented or not. This is distinct from a mere list of data or instructions or a way of viewing entropy. Active information has been used in a number of papers relative to the mind/matter relationship (Hiley, 2001 and Hiley \& Pylkkänen, 2005). The colloquial understanding of information is that it is data from which meaning can be extracted by an intelligent entity. Hiley regards it as a fundamental question as to whether information has objective significance devoid of the subjective involvement. Verbal communication is seen as a particular problem, where meaning is translated into sound waves and then back into meaning. Hiley relates this 
meaning to the agency of the speaker and the agency of the listener. He relates this inseparable link to Bohr's notion of the indivisibility of the quantum action, which cannot distinguish between the system under observation and the means of observation.

Thus, in our cosmological model we can say that the requirements of the well-definiteness of the pseudo-tachyonic part of the Hamiltonian operator in the Wheeler-DeWitt equation, does not reveal the effect of the quantum avoidance of the cosmological singularity. Hence, there some kind of classical-quantum correspondence, as it was shown in the classical tachyon and pseudo-tachyon model exists an infinite one parameter set of the cosmological evolution. Which implied an undivided wholeness of the universe and an implicate order, from which arose the explicate order of the universe as we actually experience it. The explicate order is seen as a particular case of the implicate order. The concept of implicate order of David Bohm 1980, 1990, in which particles and their more complex forms in our classical world are steered by, so called, pilot waves that operate from a 4-dimensional hidden domain, in a mode of active information. The implicate order applies both to matter and consciousness, and it can therefore explain the relationship between these two apparently different things. Mind and matter are here seen as related projections into our explicate order from the underlying reality of the implicate order. Bohm claims that when we look at the extension of matter and separation of its parts in space, we can see nothing in these concepts that helps us with understanding consciousness.

We believe that the quantum potential could be extracted from the previous energy stages (first stage) with $\mathrm{SU}(6), \ldots$..etc. then to the present of the second stage with $\mathrm{SU}(5)$ by the process of energy transfer through the Wheeler-DeWitt wave functions and that quantum potential could act as an information potential. In transmitting a signal there is a trade off between the duration of the pulse and the frequency. There is an ambiguity in the signal that is similar to the uncertainty in quantum mechanics. Hiley refers it, to the two-slit experiment, where the potential is claimed to cover the whole experimental arrangement. The quantum information changes in relation to any change in the experimental arrangement, and this is related to information entering the brain and changing the arrangement of its parts.

Again within the brain, Bohm thought that meaning was in the process itself. Bohm proposed that there were two sides or two poles to the brain, the manifest or relatively stable material side and other is the subtle mind-like side. The manifest side is classical physics, while the subtle side is the quantum level that produces the classical level. Thus the mind cannot be separated from matter. The ambiguity or uncertainty of the quantum comes through in the ambiguity attached to meaning. The quantum is seen as a pool of information shared by entangled particles. I think that the information potential may be gained by the quanta-like energies with exchanging the boson particles from $\mathrm{SU}(6)$ to $\mathrm{SU}(5)$ or vice-versa. That means when the potential or pool vanishes (according to our perception), the classical world emerges. Hiley also agrees that this system could operate in terms of quantum fields. But the description seems to be the lack of detail as to how the quantum mechanism would operate in the brain. The emergence of meaning could be developed from the theory of new energy sources $\mathrm{SU}(6), \mathrm{SU}(12), \ldots$ etc., the new concepts about the mechanisms of consciousness. It is clear from the Bohm's view, that all the separate objects, entities, structures, and events in the visible or explicate world around us are relatively autonomous, stable, and temporary 'sub-totalities' derived from a deeper, implicate order of unbroken wholeness. Bohm gives the analogy of a flowing stream: On this stream, one may see an ever-changing pattern of vortices, ripples, waves, splashes, etc., which evidently have no independent existence as such. Rather, they are abstracted from the flowing movement, arising and vanishing in the total process of the flow. Such transitory subsistence as may be possessed by these abstracted forms implies only a relative independence or autonomy of behaviour, rather than absolutely independent existence as ultimate substances. Bohm tried to adopt an interpretation of the wave as a manifestation of a deeper level, perhaps associated with consciousness. Also from the Pilot-wave theory of Antony Valentini follows quantum mathematics, but at the same time allows for new possibilities beyond conventional quantum mechanics. It's a theory that says there is indeed an objective reality behind the things we observe, that quantum uncertainty is not fundamental, and that somewhere, somehow, time is universal not relative which implies to hidden variables that are objective.

American physicist John Archibald Wheeler (1990, 2002) who suspected that reality exists not because of physical particles, but rather because of the act of observing the universe. "Information may not be just what we learn about the world. It may be what makes the world". In other words: when humans ask questions about nature, there is an active transfer of information in the domain of quantum waves where, in principle, backward causation from the future is possible. The second arrow (from future to past) remain hidden (unnoticed) for us, because life is trapped in the momentum of time. Entanglement means that particles separated at any distance, under certain conditions, can have mutually determined properties (are correlated). Hence universe multiple paths or life lines are laid out of which the individual chooses a single one, i.e. there exists a moment of grand super unified theory $\mathrm{SU}(11)[\supset \mathrm{SU}(5) \times \mathrm{SU}(6) \times \mathrm{U}(1)]$ coming after an infinite numbers of such symmetry breakings. Such non-locality becomes manifested by observation (or collapsed of the wave functions), as has been shown by electron spin orientation or polarized light. This might also be viewed as backward causation. 
According to Wheeler's (1990) and Feynman's electrodynamics, emitters coincide with retarded fields, which propagate into the future, while absorbers coincide with advanced fields, which propagate backward in time. This time-symmetric model leads to predictions identical with those of conventional electrodynamics. For this reason it is impossible to distinguish between time symmetric results and conventional results. Explanation of John Cramer (1988), Transactional Interpretations of Quantum Mechanics the transaction between retarded waves, coming from the past, and advanced waves, coming from the future, gives birth to a quantum entity with dual properties of the wave/particle. Thus the wave property is a consequence of the interference between retarded and advanced waves, and the particle property is a consequence of the point in space where the transaction takes place".

The principle of backward causation has been experimentally demonstrated recently. Aharonov's team and various collaborating groups (see Aharonov, 2010), studied whether the future may influence the past by sophisticated quantum physics technology. Aharonov concluded that a particle's past does not contain enough information to fully predict its fate, but he wondered, if the information is not in its past, where could it be? Clearly, something else must also regulate the particle's behaviour. Thus we may explain that the future may be influenced by the past by introducing some new energy sources as $\mathrm{SU}(6), \mathrm{SU}(12), \ldots$ etc. called pseudo energy groups, which controls the future particles carrying with information's. We assume that our existing 4dimensional universe is actually unfolded from 10-dimensional space-time/with the symmetry breaking of $\mathrm{SU}(11)$ of the super-unified group, although our matter universe or so called real/physical universe, unfolded from the symmetry breaking of the unified group SU(5). The first stages always remained in the form of waves and the boson particles of SU(6) are so strong, that the quanta-like is transferred through the wave functions collapses or transferred into quanta particles of the unified Gaussian energy group SU(5), that means the exotic matter fluid is transferred into ordinary matter fluids where the energy is in the form maintaining the waveparticle duality principle, the entanglement. So, between two particles within the standard model of physics there must be existence a pseudo-energy force, carrying with information energies through the waves. We view quantum particle as entangled, i.e. maintaining the wave particle duality principle. Hence it is possible, that the same quantum particles occurred in different places, due to the existences of latent-particles formed by SU(6) between two places that means our universe always is filled with some new energies. According to Stapp, 2009, 2012 , the brain is viewed as a self-programming computer with self-sustaining neural patterns as the codes. It is necessary to integrate the code from sensory input, with the code from previous experience. This creates a number of probabilities, from which consciousness has to select. The conscious act is the selection of a piece of top-level code, which then exercises control over the flow of neural excitation. The unity of conscious thought comes from a unifying force in the conscious act itself. It selects a single code from amongst a multitude on offer in the brain. This is suggested to close the traditional explanatory gap between thought and classical physics, because here the conscious thought is the selection of the code that allows the physical act, which proves the signature of the existences of the energy groups $\mathrm{SU}(6), \mathrm{SU}(12), \mathrm{SU}(24), \ldots$ etc and a relations between all energy groups by commanding from top-level to bottom-level. According to Stapp, top- level codes instruct lower-level codes to produce new top level codes and to initiate their storage in memory. There are close connections between the top-level code and the memory structure. The lower level codes have to be functioning correctly i.e. not damaged, and to be focused on the incoming stimuli in order for it to be put into higher level code and to be registered in memory. It is necessary to find the neurons providing the top level coding, then the mechanism for storing memory traces of this, and finally the mechanism by which these memories are involved in the production of new top-level codes. Stapp attaches great importance to the idea of the formation of a record. This is seen as analogous to the Geiger counter that registers a record of a quantum event. Every conscious experience is seen as recordable, because it is evidence of some form of brain process.

Again the identity theory of mind claims that each mental state is identical to some process in the brain. However, classical physics says that the entire causal structure of a physical system is determined by the microscopic level of the physical structure, so that larger scale effects such as consciousness cannot have any influence. A potential problem with the whole Copenhagen influenced interpretation of quantum theory is its possible dualism. Mathematics can be seen as a mental process instantiated in protein, which, in principle, cannot directly influence the external world. Somehow the mathematical description of the quantum waves is sitting out there in space, and then as a result of a measurement becomes a physical particle. In Copenhagen, a mental concept external to the body seems to become physical with no explanation as to how the two could interact. The Copenhagen system has the additional problem of what was happening before human minds emerged to perform measurements, for which Stapp's explanation appears rather sketchy. Consequently, a more detailed model is required to picture the inherent interaction between a more general of consciousness as a measuring device in evolution. Hence we required a new concept for the explanations of the present situations of our universe beyond the standard model of physics. 


\section{Difference Between Computer Robot And Human Brain}

What is the actual difference between computer robot and brain of human? According to Feferman 1996 who agrees with Penrose that understanding is essential to mathematical thought that mechanics cannot share with us. That means or by considering that human thought, and in particular mathematical thought, is not achieved by the mechanical application of algorithms, but rather by trial-and-error, insight and inspiration, in a process that machines will never share with humans. The model of mathematical thought in terms of formal systems is considered to be closer to the nature of human thought, and particularly mathematical thought, than to the functioning of Turing machines. The Turing machine model would assume that given a problem, human reason would plug away, applying the same algorithm indefinitely, in the hope of finding an answer. Feferman says that it is ridiculous to think that mathematics is performed in this way. Trial-and-error reasoning, insight and inspiration, based on prior experience, but not on general rules, are seen as the basis of mathematical success. Penrose also proposes that OR based quantum computation occurs in the brain. Penrose went on to ask, what it was in the human brain that was not based on algorithms. The physical law is described by mathematics, but it is not easy to come up with a process that is not governed by algorithms. The only plausible candidate that Penrose could find was the collapse of the quantum wave function, where the choice of the position of a particle is random, and therefore not the product of an algorithm. The quantum theory repeatedly comes back to the theme that Penrose regards the quantum world and the un-collapsed wave function as having objective existence. The important discussions implied the existences of the new energy sources SU(6), SU(12), SU(23), .......... In Penrose's view, the objective reality of the quantum world allows it to play a role in consciousness. If we consider the superposition of the quantum wave function by using Wheeler-DeWitt wave equation of the whole universe instead of using Schrödinger type wave equation of our 4dimensional universe, the energy behaves like off-on mode by the quanta particles with the strong latent force $\mathrm{SU}(6)$ which collapses into quanta particles by exchanging the bosons of $\mathrm{SU}(6)$ to $\mathrm{SU}(5)$ and then breaking into $\mathrm{SU}(3), \mathrm{SU}(2), \& \mathrm{U}(1)$ randomly through a continuous process from discrete structures of the universe otherwise having objective existences of un-collapsed wave functions. In this process, it may be possible to create a human-like brain within robotic computer and then robotic computer brain behaves like as human brain when it was given some initial information/experiences. Naturally it may be possible when created a strong field force by $\mathrm{SU}(6)$ with an electromagnetic interaction between the energy groups $\mathrm{SU}(6)$ \& $\mathrm{U}(1)$ [U(1) is an electrodynamics group] in the frame-work of $\mathrm{SU}(6) \times \mathrm{U}(1)$ and together with the weak electromagnetic interaction of energy group $\mathrm{SU}(2)$ with $\mathrm{U}(1)$ in the frame-work of $\mathrm{SU}(2) \times \mathrm{U}(1)$. Continuing this process like trial and error reasoning, insight, and inspiration are based on prior experiences and till to the reasonable experiences.

I, agree with Eugene Wigner's (see Wigner, 1992) suggestion that it is consciousness that collapses the wave function, as stated by Penrose and that the collapse of a special (objective) type of wave function produces consciousness. Penrose sees consciousness as not only related to the quantum level but also to space-time. He thinks in terms of time-asymmetrical quantum gravity [I think that quantum gravity comes from the energy group $\mathrm{SU}(6)$ ], because the universe is timeasymmetric from low to high entropy. He argues that the conventional process of collapse of the wave function is time-asymmetric. He describes an experiment where light is emitted from a source and strikes a half-silvered mirror with a resulting $50 \%$ probability that the light reaches a detector and $50 \%$ that it hits a darkened wall. This experiment cannot be time-reversed, because if the original emitter now detects an incoming photon, there is not a $50 \%$ probability that it was emitted by the wall, but instead $100 \%$ that it was emitted by the other detecting/emitting device. Hameroff and Penrose, 2011, 2013 classify all the mainstream approaches to consciousness as 'classical functionalism'. Functionalism takes no account of what the brain is made of or of anything finer grained than the level of neuron-to-neuron connections. It believes that these connections could be copied in another material such as silicon, and that the resulting construct would be conscious. Hameroff thinks that consciousness arises in the dendrites of neurons that are connected by gap junctions to form 'hyper-neurons', and that these are related to the gamma synchrony. Axonal spikes and synapses are seen as making inputs to and receiving outputs from the micro tubular process as part of an interactive system. Hameroff touches on the famous Libet, 2006 experiments that demonstrated a $500 \mathrm{~ms}$ time lag between a stimulus and the perception of it entering consciousness. He points out that changes in dendrites can lead to increased synaptic activity. This is basic to ideas about learning, memory and neural correlates of consciousness. The changes in dendrites involve the number and arrangement of receptors and the arrangement of dendrite spines and dendrite-to-dendrite connections. Axon potentials or spikes have been assumed to be the main basis of consciousness, but Hammeroff suggests that there could be other candidates. We assumed it may be played by the new energy sources as stated previous. Electrodes implanted into the brain detect mainly the activity of dendrites gap junctions plus inhibitory chemical synapses. Thus the detected synchrony derives from dendrites rather than axonal spikes. 
Gamma synchronies, in the $30-70 \mathrm{~Hz}$ range, have aroused interest as possible correlates of consciousness. Gray and Singer, 1989, found coherent gamma oscillations in the brain that were dependent on visual stimulation. It was suggested that this synchrony could solve the binding problem, which is the problem of how the different inputs into the brain are bound together into a single conscious experience. It was suggested that the synchrony related the activity of a relevant assembly of neurons. Varela, 1995 noted that synchrony operated whenever the processing of spatially separated parts of the brain were brought together in consciousness practically. Gamma synchrony has been demonstrated across cortical areas, hemispheres and the sensory/motor modalities. The synchrony is involved in a range of brain activities including perception of sound, REM, dream sleep, attention, working memory, face recognition and somatic perception. Also gamma decreases during general anaesthesia and returns on waking from this. So, gamma synchrony is the best overall correlate of consciousness. Hameroff points to gap junctions as an alternative to synapses for connections between neurons. Neurons that are connected by gap junctions depolarize synchronously. Gap junctions play a more important role in the adult brain than was previously supposed. Numerous studies show that gap junctions mediate the gamma synchrony. A neuron may have many gap junction connections but not all of them are necessarily open at the same time. The opening and closing of the junctions may be regulated by the microtubules. Hameroff suggests that cells connected by gap junctions may in fact constitute a cell assembly, with the added advantage of synchronous excitation. He discussed the role of the cytoskeleton, which is seen to determine the structure, growth and function of neurons. The cytoskeleton of the dendrites is distinct both from that found in cells outside the brain and from the cytoskeleton found in the axons of neurons. Hameroff discusses the process of anesthesia which erases consciousness, but leaves many non-conscious functions intact. Anesthetic gas molecules are soluble in a lipid-like hydrophobic environment. Such areas are present in the brain in the lipid regions of cell membranes and in hydrophobic pockets within proteins. It is suggested that anesthetic gas molecules interact with amino-acid groups via London forces, altering the normal action of London forces on the conformation of protein. Hameroff discusses quantum information processing. Quantum superposition's where the quantum waves represent multiple possibilities for the state of a particle, are known to persist until quanta are either measured or naturally interact with the rest of the environment. He takes the view that the original mainstream interpretation, Copenhagen Interpretation, puts not only consciousness but the concept of reality itself outside the standard model of physics. A recent review and update of this 20-year-old theory of consciousness published in Physics of Life Reviews, 2013, persists to claim that consciousness derives from deeper level that means we may assume that it creates from the first stage of the quanta with the energy group SU(6), plays major role to the quanta of the second stage i.e. our present dissertation and hence the finer scale activities inside brain neurons. The recent discovery of quantum vibrations in "microtubules" inside brain neurons corroborates this theory. Hameroff and Penrose suggest that EEG rhythms (brain waves) also derive from deeper level of microtubule vibrations and that from a practical standpoint, treating brain microtubule vibrations could benefit a host of mental, neurological, and cognitive conditions. Orch OR was harshly criticized from its inception, as the brain was considered too "warm, wet, and noisy" for seemingly delicate quantum processes. However, evidence has now shown warm quantum coherence in plant photosynthesis, bird brain navigation, our sense of smell, \& brain microtubules.

The research group led by Anirban Bandyopadhyay, 2011, at the National Institute of Material Sciences in Tsukuba, Japan (and now at MIT), has recently discover in warm temperature, quantum vibrations in microtubules inside brain neurons suggests that EEG rhythms also derive from deeper level microtubule vibrations. In addition, work from the laboratory of Emerson and Eckenhoff et al, 2013, at the University of Pennsylvania, suggests that anesthesia, which selectively erases consciousness while sparing non-conscious brain activities, acts via microtubules in brain neurons. The origin of consciousness reflects our place in the universe, the nature of our existence. Again Hameroff explained that consciousness depends on biologically 'orchestrated' coherent quantum processes in collections of microtubules within brain neurons, that these quantum processes correlate with, and regulate, neuronal synaptic and membrane activity. The continuous Schrödinger evolution of each such process is supposed to terminate in accordance with the specific Diósi-Penrose (DP) scheme of 'objective reduction' ('OR') of the quantum state. This orchestrated OR activity ('Orch OR') is taken to result in moments of conscious awareness and/or choice. The DP form of OR is related to the fundamentals of quantum mechanics and space-time geometry, so Orch OR suggests that there is a connection between the brain's bio-molecular processes and the basic structure of the universe. The DP version of OR involves a different interpretation of the term 'quantum gravity' from what is usual. Current ideas of quantum gravity (see, for example, Smolin, 2004), normally refers, instead, to some sort of physical scheme that is to be formulated within the bounds of standard quantum field theory-hence it is needed a new concept of the quantum field 
theory to explain the actual occurrence about the origin of consciousness and such explanation may be found-out from the theory of my new energy sources of $\mathrm{SU}(6), \mathrm{SU}(12), \mathrm{SU}(23), \ldots \ldots \ldots \ldots$. It is helpful to have a conceptual picture of quantum superposition in a super gravitational context that means in the super-unified theory of $\mathrm{SU}(11)[\supset \mathrm{SU}(5) \times \mathrm{SU}(6) \times \mathrm{U}(1)]$, where the energy group $\mathrm{SU}(6)$ creates gravitational field. According to modern accepted physical theories, reality is rooted in 3-dimensional space and a 1-dimensional time, combined together into a 4-dimensional space-time. But here we consider that the origin of reality is rooted in $(4+\mathrm{D})$-dimensional Friedmann-Robertson-Walker type universe having complex scale factor $\mathbf{R}+i \mathbf{R}_{\mathbf{I}}$, where $\mathbf{R}$ is the scale factor corresponding to the usual 4-dimensional Universe while $\mathbf{R}_{\mathbf{I}}$ is that of $\mathrm{D}$-dimensional space. It is then compared with $(4+\mathrm{D})$-dimensional Kaluza-Klein cosmology having scale factors $\mathbf{R}$ and $\mathbf{a}\left(=\mathbf{i} \mathbf{R}_{\mathrm{I}}\right)$, the internal space-time. It is shown that the rate of compactification of higher dimension depends on extra dimension ' $\mathrm{D}$ '. The Wheeler-DeWitt equation is constructed and general solution \& particular solution is obtained. It is found that for D $=6$ (i.e. in $4+6=10$ dimension), the Wheeler-DeWitt equation is symmetric under the exchange $\mathbf{R}_{\mathbf{I}} \leftrightarrow \mathbf{R}$. Hence, according to the new theories our actual reality of the so called material universe is rooted in 10-dimensional space-time instead of 4-dimensional space-time. Our conscious energies are associated with 10-dimensional to 5-dimensional space-time. The initial part of each space-time is at the upper left of each individual space-time diagram, and so the bifurcating space-time diagram on right moving downward and rightward illustrates two alternative mass distributions evolving in time, their space-time curvature separation increasing mechanically (so long as OR has not taken place), the 'physical reality' of this situation, as provided by the evolving wave function, is being illustrated as an actual superposition of these two differing space-time manifolds, The OR process is considered to occur when quantum superposition's between such slightly differing space-times take place differing from one another by an integrated space-time measure.

In the situations under consideration here, where we expect a conscious brain to be at far from zero temperature, and because technological quantum computers require zero temperature, it is very reasonable to question quantum brain activities. Nevertheless, it is now well known that superconductivity and other largescale quantum effects can actually occur at temperatures very far from absolute zero. Indeed, biology appears to have evolved thermal mechanisms to promote quantum coherence. Ouyang and Awschalom, 2003 showed that quantum spin transfer through phenyl ring $\pi$ orbital resonance clouds (the same as those in protein hydrophobic regions, are enhanced at increasingly warm temperatures. Spin flip currents through microtubule pathways, may be directly analogous). In the past 6 years, evidence has accumulated that plants routinely use quantum coherent electron transport at ambient temperatures in photosynthesis Engel et al, 2007 and Hildner, 2013. Photons are absorbed in one region of a photosynthetic protein complex, and their energy is conveyed by electronic excitations through the protein to another region to be converted to chemical energy to make food. In this transfer, electrons utilize multiple pathways simultaneously, through $\pi$ electron clouds in a series of chromophores (analogous to hydrophobic regions) spaced nanometres apart, maximizing efficiency (e.g. via socalled 'exciton hopping'). Chromophores in photosynthesis proteins appear to enable electron quantum conductance precisely like aromatic rings are proposed in Orch OR to function in tubulin and microtubules.

Quantum conductance through photosynthesis protein is enhanced by mechanical vibration, and microtubules appear to have their own set of mechanical vibrations e.g. in megahertz as suggested by Sahu et al., 2013. Megahertz mechanical vibrations is ultrasound, and brief, low intensity (sub-thermal) ultrasound administered through the skull to the brain modulates electrophysiology, behaviour and affect, e.g. improved mood in patients suffering from chronic pain, perhaps by direct excitation of brain microtubules. Bandyopadhyay's experiments do seem to provide clear evidence for coherent microtubule quantum states at brain temperature. The Hameroff-Penrose form of orchestrated objective reduction (Hameroff and Penrose, $2011,2013)$ is related to the fundamentals of quantum mechanics and space-time geometry that means a connection between the basic structure of the Universe and bio-molecular processes. Relating these effects to neurons might appear an unjustified self-inflicted limitation and, in this perspective, the general conclusion should not be avoided: consciousness is a property and a manifestation of life, life is universal in principle. Thus, consciousness is in principle universal". A note of caution: Roger Penrose himself recently said: "I do not see why we should take quantum mechanics as sacrosanct. I think there were going to be something else which replaces it". These words, if they can be considered as not being out-of-context, find their explanation in the incompleteness of quantum theory. For the completeness, we should consider the extension of the quantum theory with the new energy groups $\mathrm{SU}(6), \mathrm{SU}(11), \mathrm{SU}(12), \mathrm{SU}(23), \ldots \ldots \ldots \infty$ other than the unified group $\mathrm{SU}(5)$, appeared by the symmetry breaking from infinite. The last destination of the process of symmetry breaking is to be unified energy group $\mathrm{SU}(5)\left[\supset \mathrm{SU}(3) \times \mathrm{SU}(2){ }_{\mathrm{L}} \times \mathrm{U}(1)\right]$, the standard model of physics according to our present assumption. The quanta of the super-unified group $\mathrm{SU}(11)$ breaks into $\mathrm{SU}(6) \times \mathrm{SU}(5) \times \mathrm{U}(1)$, in which from 10-dimensional to 5-dimensional spacetime plays a vital role creating as consciousness with conscious particles of atom-like or element- 
like by the strong force $\mathrm{SU}(6)$. In respect to $\mathrm{SU}(5)$, where quark with charge particles forms atoms of the elements and compound elements also bio-molecules etc. of the material world instructed by conscious energy and then consciousness arrives within the complete living body. There are created two types of electromagnetic interaction in the frame-work $\mathrm{SU}(6) \times \mathrm{U}(1)$, which are also responsible for conscious sensory or intelligence with respect to the ordinary sensory with the frame-work $S U(2)_{L} \times U(1)$. Consciousness seems to be folded till to the symmetry breaking of $\mathrm{SU}(5)$ or otherwise the creation of live-element i.e. till to the 4-dimensional space-time and then unfolded. Anirban Bandyopadhyay and his colleagues from 2009, at the National Institute of Material Sciences in Tsukuba, Japan, were able to use nanotechnology to address electronic and optical properties of individual microtubules (Sahu et al, 2013a, b). The group has made a series of remarkable discoveries suggesting that quantum effects do occur in microtubules at biological temperatures. First, they found that electronic conductance along microtubules, normally extremely good insulators, becomes exceedingly high, approaching quantum conductance, at certain specific resonance frequencies of applied alternating current (AC) stimulation. These resonances occur in gigahertz, megahertz and kilohertz ranges, and are particularly prominent in low megahertz (e.g. $8.9 \mathrm{MHz}$ ). Conductance's induced by specific (e.g. megahertz) AC frequencies appear to follow several types of pathways through the microtubule - helical, linear along the microtubule axis, and 'blanketlike' along/around the entire microtubule surface. Second, using various techniques, the Bandyopadhyay group also determined $\mathrm{AC}$ conductance through 25 -nm-wide microtubules is greater than through single 4-nm-wide tubulins, indicating cooperative, possibly quantum coherent effects throughout the microtubule, and that the electronic properties of microtubules are programmed within each tubulin. Their results also showed that conductance increased with microtubule length, indicative of quantum mechanisms. The resonance conductance ('Bandyopadhyay coherence' -'BC') through tubulins and microtubules is consistent with the intra-tubulin aromatic ring pathways, which can support Orch OR quantum dipoles, and in which anaesthetics bind, apparently to selectively erase consciousness. Bandyopadhyay's experiments do seem to provide clear evidence for coherent microtubule quantum states at brain temperature. So the fact for instance that anaesthetic gas exert their effects on consciousness, and that actual evidence from genomics and proteomics point to anaesthetic action in microtubules. Frohlich saw the electric potential across the cell membrane as the macroscopic observable of an underlying quantum order. Frohlich's studies claim to show that with oscillating electrical charges in a thermal bath, a large number of quanta may become condensed into a single state, known as a Bose condensate, allowing long-range correlations amongst the dipoles involved. He also proposed that bio-molecules with a high electric dipole moment line up along the actins filaments, and that electric dipole oscillations propagate along these filaments in the form of quantum coherent waves. There is some support for these ideas, in the form of experimental confirmation that bio-molecules with high electric dipole moment have a periodic oscillation (Gray and Singer, 1989). The hypothesis of an individual double as created by our mind Vitiello agrees with Frohlich in arguing that living system constitute ordered chains of chemical reactions, which could normally be expected to collapse in the random chemical environment of biological tissue. In Vitiello's view stable ordering comes from the quantum level, but this is described by quantum field theory rather than quantum mechanics. He also claims that the folding of protein, which is fundamental to the activity of cells, cannot be described by classical physics, but could be quantum ordered. Vitiello, 1995, 2001provides citations, which he feels support a quantum dynamical view of biological tissue, notably studies of radiation effects on cell growth ,on electromagnetic fields and stress, on dynamical response to external stimuli, on non-linear tunnelling, on coherent nuclear motion in 72 membrane proteins, on optical coherence in biological systems, on weak radiation fields and biological systems by (Popp, 1986) and on energy transfer via solitons and coherent excitations. QBD proposes that the cortical field not only interacts with, but also to a good extent controls the neuronal network. It suggests that bio-molecular waves propagate along the actin filaments, an important part of the cytoskeleton, particularly in the vicinity of the cell membrane and dendritic spines. The waves derive energy from ATP molecules stored in the membrane, and these in turn are controlled by calcium ions. These waves are also suggested to control the action of ion channels, which are crucial in the transmission of signals to the synapses. The neurons membrane is further suggested to act as a Josephson junction providing insulation between two layers of superconductivity. The superconductivity current across the membrane can be controlled by the electrical potentials across the same membrane. Vitiello also discusses the question of quantum de-coherence. He claims that QBD only requires quantum oscillations to last 10-14 picoseconds, which should be much shorter than the period required for de-coherence (Del Giudice, 1988, 2002). In common with Stuart Hameroff, he additionally argues that ordered water around protein molecules may shield them from the surrounding thermal bath.

The concepts derive from the Japanese physicist, Hiroomi Umezawa1993 who speculated that understanding the processes of memory in the brain would involve quantum field theory. This led onto the idea that understanding consciousness would also involve quantum field theory. Meijer and Simon Raggett discuss the dendritic network at length. They point out that it is much more sophisticated than the axonal network. The 
dendritic membrane comprises bio-molecules with electric dipoles the positive poles of the membrane are aligned on the inner surface and the negative poles on the outer surface. The negative poles on the outer surface attract positive ions, while the positive poles on the inner surface attract negative ions. The regions where these interactions occur are called Debye layers. The dendrites of several neurons are often entangled in a network. Chemical synapses are located on the tips of dendritic spines and there are emphases on the dendritic membranes. In such processes even quantum tunnelling may play a significant role. Since the 1970s, Evan Harris Walker has proposed that quantum tunnelling of electrons would take place across junctions between Neurons. Stuart Hameroff says that "... Gap junctions enable quantum tunnelling among dendrites ...".According to the principles of modern physics: if a particle such as an electron encounters a barrier such as the synaptic junction, there is a finite probability that the particle will ... be found on the other side. From the point of view of Bohm's pilot wave quantum theory, Peter R. Holland says that quantum tunnelling is explained because the effective barrier potential is not the classical barrier potential, but is the quantum potential. There is experimental confirmation that bio-molecules of high electric dipole moment have a periodic oscillation (Fröhlich, 1968) and suggest that these oscillations are crucial to the functioning of the brain. This can be called wave cybernetics, because the wave or bio-molecule oscillation is seen as the controlling factor in the brain. Frohlich proposed a theory where bio-molecules with high electric dipole moment line up along the actin filaments immediately below the cell membrane, while electric dipole oscillations propagate along each filament as coherent waves. These are maintained by electrons trapped in and moving along the protein molecules. This is now known as a Frohlich wave. These waves exchange energy with the electromagnetic field. Stuart, Umezawa, and Takahashi, 1978 proposed the idea of a cortical field. This interacts with the macroscopic dynamics of the main neural network, which in turn transmits signals to the body tissues. The filamentous strings found in the cells also extend outside the cells forming an extra-cellular matrix that is also linked to the cell membrane. So the membrane proteins are linked both to the cytoskeleton and the extra-cellular matrix. Meijer and Simon Raggett propose that Fröhlich waves propagate along the filamentous strings. The waves are produced by energy stored in ATP molecules at membrane protein sites, which are in turn controlled by calcium ions. The waves also effect the operation of ion channels, which control neural impulses. Also suggest that this structure can give rise to macroscopic quantum phenomena, similar to superconductivity. They also regard the cell membrane as an insulating layer between two areas of superconductivity, otherwise known as a Josephson junction. This means that superconductivity current across the Josephson junction can be controlled by electric potential differences in the insulating layer. He suggests that this quantum activity may facilitate the functioning of the brain and in particular an interface between the proposed cortical field and the neurons network. The cortical field is proposed to contain energy quanta behaving as particles, called corticons. Corticons are suggested to exist everywhere in the cerebral cortex. The interface between the cortical field and the neuron network takes place in the waves propagating along the filamentous strings in the cytoskeleton and the extracellular matrix.

McFadden starts by stating that synchronous firing in the brain correlates with awareness and perception indicating that disturbances in the brain's electromagnetic field also correlate with these. This field is a representation of neuronal information and its dynamics could be seen as a correlate of consciousness. McFadden, 2001views this field as the physical substrata of consciousness. The brain's electromagnetic field is induced by neuron firing, and also the movement of ions involved in the fluctuation of electrical potential along the cell membrane. The structure of the cortex tends to amplify the induced field. Experiments in the olfactory bulb have demonstrated EEG activity in response to sensory stimuli. Information about the stimuli related to the spatial pattern of the EEG amplitude.

We conclude that the brain contains a highly structured extra-cellular electromagnetic field. The field is weak with the trans-membrane fields being about 3,000 times stronger. It is suggested that neurotransmission through gap junctions may be voltage dependent and therefore sensitive to local fields. However, McFadden prefers to concentrate on the voltage-gated ion channels in the cell membranes, because their role is better understood. Synchonous firing is thought to due to a large number of spatially distributed neurons. It is thought that many millions of neurons could be influenced by such firing. McFadden claims evidence for neuron communication via the electromagnetic field. The electromagnetic field theory of consciousness is a part of the integral electromagnetic spectrums. The medical use of transcranial magnetic stimulation (TMS) is taken to indicate the sensitivity of the brain to weak electromagnetic fields, and as this has impacts on behaviour, it is argued to impact neuronal computation and neuronal function. Even when fields are weaker than the surrounding noise, they can modulate neurons. The brain's electromagnetic field is argued to hold the same information as the neuron firing patterns. The widespread of the electromagnetic field would help to explain the unity of consciousness. He thinks that consciousness is not actually the electromagnetic field, but its ability to transmit information to neurons. McFadden declares by fiat that one particular part of the otherwise ordinary material of the brain produces consciousness. Again, it is reasonable to say that evolution selected for a particular type of field that could fine tune the neurons, but the additional production of a feeling of free will, which is false, has no demonstrable value. 
It is very convenient to say about the participation of the neutral current occurs in the frame-work of the super-unified group $\mathrm{SU}(11)[\supset \mathrm{SU}(6) \times \mathrm{SU}(5) \times \mathrm{U}(1)$ ], i.e. specially in the frame-work $\mathrm{SU}(6) \times$ $\mathrm{U}(1)$, where $\mathrm{SU}(6)$ having 35 -bosons, out of which five bosons namely $\mathbf{J}_{\mathbf{K} 3}, \mathbf{J}_{\mathbf{K} 8}, \mathbf{J}_{\mathbf{K} 15}, \mathbf{J}_{\mathbf{K} 24}, \mathbf{J}_{\mathbf{K} 35}$, corresponding to the five diagonal matrices, thinks to plays a key role for the consciousness of the universe also for human consciousness. The strong neutral current created by $\mathrm{SU}(6)$ with $\mathrm{U}(1)$ are likely to be compared with the weak interaction of $\mathrm{SU}(2)$ with $\mathrm{U}(1)$ of the unified group $\mathrm{SU}(5)[\supset \mathrm{SU}(3) \times \mathrm{SU}(2) \times \mathrm{U}(1)]$, where $\mathrm{SU}(2)$ does not directly involve with electric, it still seems to demand charged bosons $\mathbf{W}_{\mathbf{1}}$ and $\mathbf{W}_{\mathbf{2}}$. This circumstances prompted efforts to link it with an electromagnetic interaction. This link achieved via $\mathrm{SU}(2) \times \mathrm{U}(1)$ frame-work originally proposed by A. Salam and S. Weinberg and sometimes called the electro-weak interaction. The link brings the photon (which is a boson), closer to three particles $\mathbf{W}_{\mathbf{1}}, \mathbf{W}_{\mathbf{2}} \& \mathbf{W}_{\mathbf{3}}$, where $\mathbf{W}_{\mathbf{1}}, \mathbf{W}_{\mathbf{2}}$ are two opposite charged particles and the third $\left(\mathbf{W}_{3}\right)$ neutral. In this unified picture it is more convenient to say that another neutral particle $\mathbf{Z}^{\mathbf{0}}$ instead of $\mathbf{W}_{3}, \mathbf{Z}^{\mathbf{0}}$ has zero mass and charge, just like the photon. However, the photon does not interact with the neutrino while the $\mathbf{Z}^{0}$ does. The exchange of $\mathbf{Z}^{\mathbf{0}}$ does not alter electric charge, and hence such an interaction is called a neutral current interaction. Similarly, if we go beyond the standard model of physics i.e. in the symmetry theory of SU(11), we see there are five neutral particles of the latent energy group $\mathrm{SU}(6)$, in which two pairs, namely $\mathbf{J}_{\mathbf{K} 3}, \mathbf{J}_{\mathbf{K} 8}$ and $\mathbf{J}_{\mathbf{K} 15}, \mathbf{J}_{\mathbf{K} 24}$ were interchanged without any colour changes, but the neutral particle $\mathbf{J}_{\mathbf{K} 35}$, like as $\mathbf{Z}^{\mathbf{0}}$ also create a neutral current i.e. electromagnetic interaction through the frame-work $\mathrm{SU}(6) \times \mathrm{U}(1)$, called pseudo-electromagnetic interaction which may responsible for the consciousness sensory with material weak electromagnetic interaction achieved by the frame-work of $\mathrm{SU}(2) \times \mathrm{U}(1)$. We observe that, towards unification of $\mathrm{SU}(3), \mathrm{SU}(2), \mathrm{U}(1)$, the strength of weak force gradually increases while strength of strong force gradually decreases and ultimately we found the unified group SU(5). So, in the theory of SU(11) i.e. in another phase we found quark-like \& lepton-like particles, which may be five times of each quark [ i.e. u-quark, d-quark,.....etc. of the standard model of physics of the unified group $\mathrm{SU}(5)]$ or each of five different quarks [namely, $\left\{\boldsymbol{u}_{\mathbf{1}}, \boldsymbol{u}_{\mathbf{2}}, \ldots, \boldsymbol{u}_{\mathbf{5}}\right\}$-quarks, $\left\{\boldsymbol{d}_{\mathbf{1}}, \boldsymbol{d}_{\mathbf{2}}, \ldots ., \boldsymbol{d}_{5}\right\}$ quarks,...etc.]. Thus $\mathbf{Z}^{\mathbf{0}}$ - like neutral particle of SU(6) like zero mass \& charges instead of $\boldsymbol{J}_{\boldsymbol{K} 35}$ interact with other particles of $\mathrm{SU}(5)$ creating strong neutral current with conscious sensory information system.

In the theoretical physics, we observe the flavour-changing neutral current (FCNC's) are hypothetical expressions that change the flavour of a fermions current without altering its electric charge. If they occur in nature (as reflected by Lagrangian interaction terms) these processes may induce phenomena that have not yet been observed in experiment. Flavour-changing neutral currents may occur in the standard model beyond the tree level, but they are highly suppressed by the GIM mechanism. FCNC's are generally predicted by theories that attempt to go beyond the standard model, such as the models of Super-Symmetry or Technicolor. Their suppression is necessary for an agreement with observations, making FCNC's important in model-building. The Cemi-field theory conceives that the electromagnetic field in the brain fine tunes the probabilities of neuron firings. The affected neurons may be a part of the larger connected assemblies, and this leads to memory and learning. In simulated networks non-synaptic neuronal interactions via the electromagnetic field and also gap junctions enhance learning. Modulation of long term potentiating by electromagnetic fields has also been demonstrated in vitro in rat hippocampus slices.

Consciousness was a fundamental property of electrical charge, or individual charged particles, or the photons that intermediate it, thus making it a primitive or a brute fact of the universe. Thus we can say that the perception indicating that disturbance in the brain correlates with pseudo-electromagnetic field. The brain's pseudo-electromagnetic field is induced by neuron firing, and also the movement of ions involved in the fluctuation of electrical potential along the cell membrane. The structure of the cortex tends to amplify the induced field. Experiments in the olfactory bulb have demonstrated EEG activity in response to sensory stimuli. Information about the stimuli related to the spatial pattern of the EEG amplitude. Brain contains a highly structured extra-cellular electromagnetic field. The field is weak with the trans-membrane fields being about 3,000 times stronger. It is suggested that neurotransmission through gap junctions may be voltage dependent and therefore sensitive to local fields. Synchronous firing is thought to due to a large number of spatially distributed neurons. It is thought that many millions of neurons could be influenced by such firing. McFadden claims evidence for neuron communication via the electromagnetic field. The medical use of trans-cranial magnetic stimulation (TMS) is taken to indicate the sensitivity of the brain to weak electromagnetic fields, and as this has impacts on behaviour, it is argued to impact neuronal computation and neuronal function. Even when fields are weaker than the surrounding noise, they can modulate neurons. The brain's electromagnetic field is argued to hold the same information as the neuron firing patterns. The widespread of the electromagnetic field would help to explain the unity of consciousness. The high conductivity of the cerebral fluid in the brain ventricles makes the brain into a kind of Faraday's cage, insulating it from external electrical fields. However, it is much easier for magnetic fields to penetrate the brain and other tissues. Moving magnetic fields such as those used in TMS do produce effects in the brain. 


\section{Role Of Dendrites For Consciousness}

Romijn views the brain as a chaotic self-organising process, the outcome of which is the pattern of electric and magnetic fields generated by the dendritic trees of neurons. Virtual photons comprise the electric and magnetic fields and it is these which are claimed to encode conscious experience. Romijn argues that they are causally necessary and sufficient for consciousness. He takes an initially conventional approach in pointing out that brain scan studies show a correlation between neural activity and subjective experiences (Raichle, 1998; Schacter et al, 1998). Thus he takes the view that subjective experience is as real for the brain scan activity is for the third party investigator.

Romijn discusses the detailed behaviour of dendrites. When a dendrite receives a signal from another neuron there is a depolarisation of the membrane, in the case of an excitatory signal, and hyperpolarisation in the event of an inhibitory signal. This creates an electric field between the part of the dendrite membrane that has become polarised or hyperpolarised and the rest of the membrane. The greater part of the electric field will flow towards the cell body and the axon hillock because the dendrite is thicker in that direction. This action along the dendrite also generates a magnetic field. The dendritic tree has been shown to use several different forms of information processing. At the synapse the incoming pattern of action potentials determines which of various neurotransmitters stored there are released. On the dendritic side, receptors are sensitive to particular neurotransmitters. The receptors are clustered in complex spatial patterns. Receptors can modulate each other performance. Outside the synaptic cleft, the extra-cellular fluid has a low concentration of ions, neurotransmitters and hormones that may exert a synchronising effect between neurons. Studies have shown that the dendritic tree can detect the individual discharge of synapses and has mechanisms for amplifying the signal to noise ratio. Dendritic spines provide the postsynaptic contact sites for $80 \%$ of excitatory synapses. Spines can change their shape in a period of milliseconds, which changes the flow of information into the dendrites.

Romijn points out that synaptic transmission are probabilistic. When an action potential reaches a synapse, there is no certainty that the synapse will fire. There is only a probability, of between $30 \%$ and $80 \%$, depending on the type of synapse that it will fire. In some studies (Lehman et al, 1998; Zeki \& Bartels, 1998), field configurations, which had to remain stable in the cortex for a minimal time such as $120 \mathrm{~ms}$ were related to various types of mental activity. Shorter lived fields are thought to relate to the unconscious level. The electrical and magnetic fields are seen as having a vast number of possible semi-stable configurations they can take up in response to either external stimuli or existing memories (Sakai \& Miyashita, 1994) and Tononi et al, 2008). These fields formed out of virtual photons, the intermediating particle/waves of the electromagnetic force, are deemed to be the carriers of consciousness in the brain.

Protein molecules constitute ion channels, receptors and enzymes and have electrically charged groups held together by vander Waals forces. These electrostatic binding forces determine the tertiary structure of proteins, and hence some of their properties. The ions, receptors and enzymes experience fluctuations as a result of the electrical field around the dendrite (Fröhlich, 1975, Goodman et al, 1995). It has been shown that postsynaptic receptor and ion distribution continually undergoes non-linear changes because of the synaptic electric fields. All this means that the dendritic tree has the ability to tune itself to the inflow of information, which in turn results in ordered electric and magnetic fields.

\section{Ion Channel Coherence Of Gustav Bernroider}

Ion channels are a crucial component in the axonal spiking/synaptic firing model of neuronal signalling and information processing. The axonal signal starts from the body of the neuron and proceeds down an extension called the axon, by means of a fluctuation in the difference in electrical potential across the membrane that forms the exterior of the axon. The membrane is formed by a double layer of lipids. The ion channels consist of protein molecules inserted through the lipid bi-layer. The axon fires when sodium $\left(\mathrm{Na}^{+}\right)$ions flow in through one set of ion channels, and subsequently returns to its resting state when potassium $\left(\mathbf{K}^{+}\right)$ions flow out through another set of ion channels. This process continues down the length of the axon until it reaches the synapse which it allows to fire, and thus communicates with other neurons. Ion channels are thus a key mechanism in the brain's signalling and information processing. Bernroider and Roy's calculations claim that ion permeation can only be understood at the quantum level. Their calculations suggest that the $\mathbf{K}^{+}$ions and the carboxyl atoms of the binding pockets are two quantum-entangled sub-systems, and they equate this to a quantum computational mapping. In a later paper, presented at the Quantum Mind conference, Bernroider, 2007, proposed that different ion channels could be non-locally entangled, thus proposing a quantum process over an extended area of the axon. Given the importance of the ion channels in brain functioning, this model would give quantum coherence and non-locality in the axon membrane an integral role in the brain's signalling and information processing. Further to this, Bernroider and Roy have pointed out a similarity between the structure of the $\mathbf{K}^{+}$ion channel and some recent proposals for making quantum computers, in which ions are held in microscopic traps. They also propose a quantum information system in the brain that is driven by the entangled ion states in the voltage-gated ion channels. These ion channels, situated in the 
Neuron membranes are a crucial component of the conventional neuroscience description of axon spiking leading to neural transmitter release at the synapses. The ion channels allow the influx and out flux of ions from the cell driving the fluctuation of electrical potential along the axon, which in turn provides the necessary signal to the synapse. Recent progress in atomic-level spectroscopy of the membrane proteins that constitute the ion channels and the accompanying molecular dynamic simulations indicate that the organisation of the membrane proteins carries a logical coding potency, and also implies quantum entanglement within ion channels and possibly also between different ion channels. An increasing number of studies show that proteins surrounding membrane lipids are associated with the probabilistic nature of the gating of the ion channels. Bernroider's theory might be seen to represent even more of a challenge to conventional neuroscience than the other quantum consciousness theories. This is because its recruits as its basis the axon membrane and ion channels which form a crucial part of the conventional neuroscience model, and then tries to remodel these core structures on a quantum-driven basis. It is hard to deny that if this theory were to become better substantiated, it would produce in neuroscience a revolution of the most profound kind. The interaction between ions, attracted water molecules and neighbouring oxygen atoms is considered to require a quantum description. This raises the question of whether quantum effects can propagate in the classical states of proteins. The access of ions to the pore gate is a relatively slow process not likely to require quantum processing. However, the selectivity filter can change its conformation from permissive to non-permissive on a much shorter timescale. It appears that in the conditions of the selectivity filter the ion's wave function can become highly delocalized over a significant part of the filter region. Bernroider's theory could potentially be a vehicle for transferring consciousness from implicate into the explicate order of David Bohm. Bernroider differs from Penrose and Hameroff's Orch OR model in his emphasis of the axons and membranes, as opposed to the dendrites and the cytoskeleton. However, there are similarities between the two models in that both of them propose quantum coherence, non-locality and subsequent wave function collapse linked to the brain's macroscopic information processing activity. As it stands, Bernroider's proposals only deal with information processing in the brain rather than consciousness as such. However, it appears possible that wave function collapse in the ion channels might link to Penrose's proposed geometry of space-time, just as readily as wave function collapse in the cytoskeleton. Chris King, 1989, 2003 2011, 2012, 2014, favours the approach of Chalmers over the approach of Dennett in looking at the problem of consciousness. .King favours the transactional interpretation of EPR type non-local quantum correlations. In his article: The transactional interpretation visualizes an exchanged particle wave function as the interference of a retarded usual time direction offer wave and a time-reversed advanced confirmation wave. Time symmetric interactions also occur in quantum field theories where special relativity allows both advanced and retarded solutions because of the energy relation $\mathbf{E}= \pm \boldsymbol{p}^{\mathbf{2}}+\boldsymbol{m}^{\mathbf{2}}$, Virtual photons and electron-positron pairs deflecting an electron in quantum electrodynamics. Since the photon is its own anti-particle, a negative energy photon travelling backwards in time is precisely a positive energy one travelling forwards. In quantum mechanics, not only are all probability paths traced in the wave function, but past and future are interconnected in a time-symmetric hand-shaking relationship, so that the final states of a wave-particle or entangled ensemble, on absorption, are boundary conditions for the interaction, just as the initial states that created them. The transactional interpretation of quantum mechanics expresses this relationship neatly in terms of offer waves from the past emitter/s and confirmation waves from the future absorbers, whose wave interference becomes the single or entangled particles passing between. When an entangled pairs are created, each knows instantaneously the state of the other and if one is found to be in a given state, e.g. of polarization or spin, the other is immediately in the complementary state no matter how far away it is in space-time. This is the spooky action at a distance, which Einstein feared because it violates local Einsteinium causality in which particles not communicating faster than the speed of light. Weak quantum measurement (Aharonov et al. 2010) is a process where a quantum wave function is not irreversibly collapsed by absorbing the particle but a small deformation is made in the wave function whose effects become apparent later when the particle is eventually absorbed e.g. on a photographic plate in a strong quantum measurement. Weak quantum measurement changes the wave function slightly mid-flight between emission and absorption, and hence before the particle meets the future absorber involved in eventual detection. A small change is induced in the wave function, e.g. by slightly altering its polarization along a given axis (Kocsis et al. 2011). This cannot be used to deduce the state of a given waveparticle at the time of measurement because the wave function is only slightly perturbed, and is not collapsed or absorbed, as in strong measurement, but one can build up a prediction statistically over many repeated quanta of the conditions at the point of weak measurement, once post-selection data is assembled after absorption. This suggests (Merali, 2010, Cho, 2011) that, in some sense, the future is determining the present, but in a way we can discover conclusively only by many repeats. Again, according to King that for good adaptive reasons, the brain goes beyond the brute force of quantum computing, to achieve intuitive decisions and creativity both of which involves subjective consciousness. These ideas appear to be similar in spirit to the Penrose concept of non-computability. In general computation seeks a single outcome while creative activity and some other behaviours, seeks diversity. Chaotic excitability is suggested as one of the earliest features of eukaryote cells. 
This would allow the single cell to get feedback from the environment, rather than becoming stuck in a particular and unsatisfactory oscillation. The behaviour of single cell organisms in being able to navigate and behave adaptively in their environment is in any case a problem for cognitive theory. The human brain also exhibits a fractal structure, that forms a suitable platform for chaotic (non-linear) reception and expression of neuronal activity.

\section{King formulates several ultimate questions:-}

a) Is there any intrinsic meaning to life, or is it we ourselves, as living beings, who express the meaning by unfolding it in our creative lives as conscious participants?

b) Is there world heading for an apocalyptic falling out and a triage of nature caused by a failure to protect our generations own futures, or is the entire universe evolving towards a state of ever-deepening realization and enlightenment?

c) Is the fulfilment of life in the universe found in some future ultimate state of enlightenment, or is it expressed eternally across space-time in the consciousness of all sentient beings which will come to witness or have witnessed the ongoing existential condition, who together bring about the historical evolution of the conscious universe?

The answers is nothing but some kind of new quantum energy transfers from the past infinite discrete universe to the present our created physical universe with splitting into spirit stuff \& physical stuff and working in the process like entanglement.

\section{Explanation About Quantum Consciousness}

Scaruffi's provides an outline of the main theories of quantum consciousness. Quantum models of consciousness date back to the 1920's and the birth of quantum theory itself. However, the earliest detailed model appears to have been produced by Evan Walker, 2000 already in 1970. This proposed that electrons could quantum tunnel between adjacent neurons to produce a virtual nervous system that direct the synapse-based system. The idea finds echoes in the later Penrose / Hameroff model, where Hameroff suggests that quantum states can extend across macroscopic areas of the brain, as a result of quantum tunnelling at gap junctions linking the dendrites of different neurons.

The physicist, Herbert Fröhlich, 1989, suggested that a quantum phenomenon called Bose-Einstein condensation could arise in biological matter. Living matter comprises mainly water and bio-molecules, both of which are electrical dipoles. It is suggested that when such oscillators are maintained at a constant temperature, as they are in the thermal non-equilibrium of biological tissue, condensates can arise. These may also encode information and transmit signals. At a later date, the Penrose/Hameroff model also used condensates, which in conjunction with quantum tunnelling at gap junctions allowed quantum states to extend over macroscopic areas of the brain. In 1989, Ian Marshall also suggested that consciousness could arise from the excitation of condensates in the brain. Also Scaruffi discusses the position of Karl Pribram, 1991, who considers that brain processes have many of the properties of holograms. Sensory perception is seen as electromagnetic activation propagating through the brain. These sensory waves can interfere with memory also propagating as a wave to produce a holographic structure. Perceptions can be analysed into component frequencies, and therefore dealt with by Fourier analyse. Dirac's least action principle constrains the trajectories of these waves.

In looking at the synapses between neurons, particular attention to the metabotropic links, as distinct from the ion tropic links that take the form of electrical signals via membrane ion channels. With the metabotropic links, neurotransmitters bind to G-protein coupled receptors (GPCR). These activate second messengers, which in turn act on protein kinesis and phosphatises that modulate the cytoskeleton. The cytoskeleton in its turn signals protein production requirements to the nucleus of the cell. The fast electrical activity of the ion channels is contrasted with the slower biochemical processes within the neuron. Georgiev says that the Hameroff model only takes account of the biochemical and not the electrical activity. He disagrees with this exclusion of electrical activity, pointing out that Penfield's ground breaking research in the mid 20th century showed that conscious memories could be evoked by inserting electrodes into parts of the cortex.

Consciousness itself must be an intrinsic physical property (Scaruffi, 2001), rather than something that is created by other physical components. He compares those neuroscientists who do not accept this, to a scientist that did not accept that electrical charge is a fundamental property, but tried to explain it in terms of some other property such as gravity. He takes the view that any paradigm that tries to manufacture consciousness out of something else is doomed to failure. In such a paradigm, consciousness will seem to appear by magic by putting neurons or similar components which have no sign of consciousness in their make up together. He says that his theory is neither dualist nor reductionist. Consciousness is seen as separate from physical science as described, but still a physical property. This approach seems close to a physical defined panpsychism. The development of molecular biology during the latter part of the 20th century made it clear that neurons were highly complex, and from this it became apparent that features such as memory and some diseases such as dementias might be better 
understood in terms of molecular changes within the neurons. In these cases, it has been shown that not only are there changes in neuronal firing, but also in cytoskeleton organization, the cytoskeleton being composed of biomolecules that are the basis of life. The DNA of the cell nucleus contains essential information, but is viewed here as being driven by the transfer of information from the cytoskeleton.

Hupin $\mathrm{Hu} \&$ Maoxin Wu, 2004, 2010, says that networks of nuclear spins in neural membranes are associated with strongly fluctuating magnetic fields that are modulated by the indirect dipole-to-dipole coupling in action potentials. Interactions between two nuclear spins result indirectly from the interactions of electrons around the nuclei. Hu and $\mathrm{Wu}$ found that these couplings had frequencies of 5-25 Hz, which are within the EEG range of activity. They predicted that magnetic fields associated with spin-related coupling across the cell membrane would have an energy value equivalent to photon emission. By Persinger et al, 2013 that the underlying spin networks could be linked to photon emissions observed with particular brain activities. The energies available to the neuronal membrane are suggested to be within the range associated with photon emission, and therefore capable of interacted with it or altering it. A recent study showed that photon emissions from cell cultures were mainly emitted from cell membranes. Another study showed that photon emissions in brain tissue varied strongly, when the tissue was either oxygen deprived, or alternatively exposed to pure oxygen. It was found that the intensity of emissions from the right hemisphere of the brain were correlated with EEG activity over the left prefrontal, when the subjects were imagining white light, but not at other times. Another study by Dotta and Persinger, 2011 looked at the behaviour of the para-hippocampalgyrei in both hemispheres, when light was imagine by the subject, there was a marked increase in power in the delta, beta and gamma bands associated with activation of the para-hippocampalgyrei. Photon emissions were elevated in the 16-17 $\mathrm{Hz}$ range, while the subject imagined light, and this corresponds to the range of proton couplings predicted by $\mathrm{Hu}$ and $\mathrm{Wu}, 2004$.

\section{Photon And Electric Charge-Mediated Consciousness}

Romijn, 1997 puts forward the concept that subjectivity or consciousness is coded into the virtual photons that generate electric and magnetic fields. This approach has considerable advantages. Photons intermediating the electromagnetic force are, as far as we know, the most basic level of the universe. At this level the fundamental components of the universe have given properties that cannot be explained, analysed or reduced further. So both the charge on the electron and the ability of the photon to intermediate it across space are given properties that cannot be explained or reduced. This is the only physical level at which it is possible to have properties that cannot be reduced to something more fundamental.

The article suggests that photons carry subjectivity or consciousness as such a given property. This is in principle possible because irreducible properties are present at this level. It is more reasonable than the mainstream approach, which suggests that a new property of consciousness can be produced by banging together previously unconscious bits of matter. The problem would be the same if we tried to say that electrical charge was a function of banging things together in some complex system. It might look plausible, but we would forever be looking for what actually happened that produced the charge. Although subjectivity is present at the level of photons, it requires brain systems to generate ordered patterns that are the basis of actual conscious experience.

The change of a larger group $\mathrm{SU}(11)$ of symmetries to the subgroup $\mathrm{SU}(6) \times \mathrm{SU}(5) \times \mathrm{U}(1)$ is spontaneous by the redistribution of energy particles of stages like gaseous as explained before. The above subgroup which contained the U(1) group, there inevitably arises particles (whose annihilation formed charge particles) that have the characteristics like a magnetic mono-pole. Typically, the mass of which (in energy units) may be $\sim \mathbf{1 0}^{19} \mathrm{Gev}$ (Plank energy). Monopoles like charge particles are highly stable particles and once created are not destructible. And so they would survive as relics to the present epoch. The explanation of the two energy groups SU(5) and SU(6) of the SUT energy group SU(11), we begin with the analogy of ferromagnetism and crucial role of the Curie-temperature $\left(\mathbf{7 7 0}^{\circ} \mathbf{C}\right.$ for iron). Above this temperature a bar of iron shows no magnetism in an external field. This is because its elementary nuclear magnets are randomly aligned with no resultant magnetization. Energetically, this is the lowest state for the bar and it chooses to remain in that state as the most stable one. Bellow the Curie temperature the state of lowest energy changes to that in which all the nuclei are aligned along the bar, which develops polarity at its ends. There are two states of the same lowest energy possible, depending on which (north or south) of the two poles falls at a given end. The ultimate choice of one state apparently breaks symmetry although theoretically and inherently the symmetry is always there. In the early universe something similar happened to the super unified theory SU(11) and then SU(5). Above like a critical temperature $\mathbf{T}_{\mathbf{c}}$, the vacuum state, the state of lowest energy, is none other than the potential $\varphi=0$. Below $\mathbf{T}_{\mathbf{c}}$, the states of lowest energy of the thermo-statistical particles are changed. It now corresponds to a situation when $\varphi$ has non-zero values. Corresponding to states of the same lowest energy, let us suppose that there exist alternative values $\boldsymbol{\varphi}_{\mathbf{i}}(\mathrm{i}=1,2,3, \ldots$.$) which now acquire that status of vacuum. There were basic$ symmetry with respect to all $\boldsymbol{\varphi}_{\mathbf{i}}$, but in practice the system may spontaneously acquire one of them. This is again 
an apparent break-down of the symmetry. The consequences of this for the very early universe are that it is divided into different domains, each with a different value of $\boldsymbol{\varphi}_{i}$. In this way the universe acquires discontinuities along the domain walls. These translate into highly significant discontinuities of matter distribution. The fact that we do not see such discontinuities in actuality (say in the form of large sheets of matter) is hard to explain away. This difficulty is known as the domain wall problem. The intersection of two domain walls is a linear structure known as "cosmic-string" such filamentary structure have been invoked in scenarios for galaxy form.

\section{Recent Experiments On Photon-Mediated Consciousness}

Recent experiments confirm Romijn's ideas in the sense that a bio-photon coherent system may constitute an ultra rapid communication system not only in the brain but also functioning in the whole organism, explaining the amazing concerted actions of complex living organisms. Regarding the biophysical visual representation idea, it should be stressed that the phrase "ultra-weak bio-photon emission" is confusing, as it suggests that ultra-weak bio-photons are not important in cellular mechanisms but are by-products of free radical reactions. In contrasts externally measured ultra-weak bio-photon emission from cells and neurons is principally produced from natural oxidation processes on the surfaces of cellular membranes as demonstrated by Blake et al. 2011.

However, the real bio-photon intensity, as produced in mitochondria, as well as DNA/RNA, can be fundamentally higher inside cells and neurons (Bókkon et al., 2010) compared with the bio-photon intensity in their surrounding environment which makes it possible for the emergence of intrinsic biophysical pictures in V1 visual areas. Long-term visual memories may not store as biophysical pictures but as red-ox regulated epigenetic codes. During visual imagery, top-down processes trigger and regulate the epigenetic encoded longterm visual information. Next, according to retrieved neural epigenetic information, mitochondrial networks in synchronized neurons generate patterns of bio-photons through red-ox reactions, which can produce intrinsic biophysical pictures in retinotopic and mitochondrial rich visual neurons during visual imagery, REMS associated dreams or visual hallucination (Bókkon, 2009; Bókkon and D'Angiulli, 2009; Bókkon et al., 2010a). First bio-photon experiments may support our biophysical pictures representation. Namely, Dotta and Persinger, (2011) and Dotta et al. (2012) observed cognitive coupling with bio-photon emission in the brain during subjective visual imagery were strongly correlated with EEG activity and the emergence of action potentials in axons. In addition, Sun et al., (2010), revealed that bio-photons can conduct along the neural fibres which supports this biophysical picture hypothesis. It appears that bio-photonic and bio-electronic activities are not independent biological processes in the nervous system, and their synergistic action may play a significant role in neural signal processes.

Basically, any charge in elementary matter, may distort, or "polarize" the quantum vacuum in the immediate vicinity, through attracting virtual particles with opposite electrical charges and repel virtual particles with similar electrical charges. In quantum field theory, the fabric of space is visualized as consisting of field, that at every point in space and time exhibit a quantum harmonic oscillator, interacting with neighbouring oscillators. Further, and also critically importantly, the wave solutions are in pairs. This means that whenever the phase arrangements of intersecting plane waves produce an electron, they will also necessarily produce the opposite phase positron next to it (they will also have opposite spin states). This explains matter-antimatter pair production, which is occurring everywhere in space all the time, because space vibrates in two distinct patterns (particles and quantum fields) that are constantly switching. Splendid work on teleportation, both in theory and experiment as reported in Nature by Sudbury (1997) and Bouwmeester et al. (1997) and in line with the work by Marcer and Schempp (1997), makes it clear that non-local quantum information represented by entanglement of particles in this field could be recovered locally as useable information.

We have from the "Noetic Field Theory" of Amoroso et al. (1999), vacuum (zero-point) quantum fluctuations and gravitation, introduced as potential mechanisms explaining non-local information exchange. So called noetic effects coupled operators of a noetic field to specific loci of pumped Frohlich-like coherent states. This was seen as a phase regulator into the patterns of Pribram's holonomic formations. The pumping mechanism for this process is inherent in the self organization of the system. The radiation pressure of the Bose states, Fermi-quasi-particle transitions, vacuum zero point fluctuations, and string dynamics are considered to be instrumental in driving this dynamic transpersonal 'memory of being'. This was supposed to be a dynamic Hilbert space storing archetypal forms of the personality or psyche. The force carrier of the electromagnetic field is the photon. At a microscopic level, therefore, the interaction between the constituent particles of matter and the quantum vacuum involves photons being exchanged between the virtual particles of the vacuum, we consider the photons belong to the energy group SU(6) created in the previous (first stage) phase, with a pseudoelectromagnetic interactions by exchanging 30-bosons of SU(6) into 30-bosons of SU(5) or vice-versa, also producing the quark-like particles which transfer into the matter particles of the group SU(5) by creating quarks, electrons etc. 
Of note, if we assume a collective storage of all information that is present and/or evolves in our Universe and that human and other intelligent species in the cosmos interact with such a knowledge field, it intrinsically implies that it cannot be solely treated as a by-product of our brain and intelligence in general. At first sight this conflicts with current mainstream science and conventional pictures of reality. However the following may show that in fact there is solid ground and even overwhelming evidence for the hypothesis/concept of a universal knowledge field. These considerations are based on the current descriptions of nature on the micro level (string and spin theories), quantum mechanical concepts (such as entanglement, nonlocality and resonance), cosmological models on energy (zero point energy and negative energy, we may consider as pseudo/latent energy explained above) as well as holographic concepts of reality and space/time modalities. In addition, universal consciousness can be approached from transcendental human experience, including transpersonal consciousness and should be discussed against a meta-physical background, also in relation to the phenomenon of consciousness and self-consciousness as well as information theory.

Penrose, 1989, proposed that spin networks could be fundamental in the description of space-time in a background lacking manner. In the brain, spin-networks were pictured as electron-unpaired electron spins that represent pixels, collectively forming a "mind screen" that is known to be highly sensitive to fluctuating internal magnetic fields and action potentials. Such perturbations were considered to modulate neural dynamics, but also could enhance synchronization and stochastic resonance as have been noticed in brain ( $\mathrm{Hu}$ and $\mathrm{Wu}, 2004)$. The particular spin chemistry bridges classical neural activity, serving as input via the magnetic influences on biochemical processing. The proper information integration, transmission and exchange with outer information domains require a guided interactive quantum process, in which the classical separation of sender and receiver is overcome through an act of measurement and/or proper resonance with the information source. This implicitly should be based on the phenomenon of entanglement and consequently on unitary and conscious field properties of the neural and exo-systems (McFadden, 2007, John, 2001, Bohm and Hiley, 1987). This allows the continuous exchange of meaningful information with global magnetic fields as proposed by McFadden, 2007 and Burke and Persinger, 2013 and/or a universal quantum knowledge field as earlier proposed by Bohm and Hiley, 1987). The implicate order concept was suggested to also contain personal information [our mental double in the universal consciousness domain, (Vitiello, 1995)]. In order to operate in a conscious as well as sub-conscious modes and also to enable modalities of self consciousness, it was proposed that apart from the known four dimensions at least one or two imaginary dimensions are required (Carter $2013 \mathrm{a}$ and b, Smythies, 2003). In my theory "The Origin of Consciousness in the Universe- IOSR" was explained that our reasonable universe are actually 10-dimensional space-time, where appeared the universe from (10 to 7) dimensional flat universe which are non-adiabatic stage, then (6 to 5)-dimensional closed universe and the stages arises are adiabatic, then our so called real/physical universe i.e. 4-dimensional Einstein's universe. Feeling of consciousness activity may associated with 6 and, or 5-dimensional space-time.

Mathematician Shor, 1996 proved a quantum error correction theorem for quantum computers. If quantum degrees of freedom in a quantum computer are de-cohering due to loss of phase information from the computer (the system) to its environment, then Shor showed that if information is added to the system from the outside, the de-cohering degrees of freedom could be made to re-cohere again. Re-coherence can occur, for instance, being driven by coherent electromagnetic field whose intensity and period distribution can be tuned non-randomly, thereby injecting information that results in a new controlled superposition state (Kauffman, 2012b). This clearly says that re-coherence is, in principle, possible. This idea finds support in the papers of physicist Briegel, 2006 showing that a quantum coherent "entangled" system can de-cohere to class city than recohere to quantum entangled coherence.

In looking for such a system, Vattay et al, 2012, examined the recent research on quantum properties of photosynthetic systems (reviewed by Arndt, 2010, Lloyd, 2012). In photosynthesis photons are captured by the chlorophyll molecule that is held by antenna protein. The chlorophyll molecule maintains quantum coherence for up to 750 femto-seconds. This is much longer than the classical prediction, and is viewed as responsible for higher than classically predicted efficiency of energy transfer. The particular antenna protein plays a role in preventing more rapid de-coherence, or in inducing re-coherence in de-cohering parts of the chlorophyll molecule. Part of the quantum system may start to de-cohere, but be forced back into coherence, described by the abovementioned quantum error correction. Kauffman thinks that this raises the possibility that webs of quantum coherence or partial coherence can extend across a large part of a neuron, and can remain poised between coherence and de-coherence.

In relating quantum coherence to consciousness, Kauffman assumes like Hameroff , that coherence would have to be sustained for the milliseconds timescales associated with neural processing, rather than the femto- and pico-second timescales associated with quantum coherence in photosynthetic organisms. The process of photon quantum teleportation (Salari et al., 2010), have been suggested for long distance signalling in the brain, a process that both employs classical and quantum elements. Ehresmann et al., 2011, stresses the dynamic character of a multi-scale flexible brain structure, varying over time and with a hierarchy of complexification 
levels, in which higher cognitive and mental processes can develop. This occurs within a 5-dimensional global landscape with retrospective and prospective elements that, among others, result in changes in the synchronization of neuronal assemblies as well as dynamic adoption of neuronal contacts. In this sense the dynamic flow of information in the brain may mirror the circular flow as it occurs from the micro-to macro scale in the whole universe and vice versa. Such a multidimensional space-time brain structure, being open to external electromagnetic and quantum fields, could also provide an interpretation framework for understanding of the, until now, non-comprehensible time delays in subconscious and conscious perception, the inner knowing of qualia as well as the subjective experience of transpersonal and extra-sensory events such as intuition, serendipity, clairvoyance and telepathy ( Libet, 2001, 2006, Jahn and Dunne, 2004).

\section{About Zero-Point Energy}

Zero-point energy is the energy that remains when all other energy is removed from a system. Zeropoint energy was experimentally demonstrated with the so called Casimir Effect, a unique attractive quantum force between closely-spaced metal plates. Casimir force was shown to be due to radiation pressure from the background electromagnetic zero-point energy which has become unbalanced due to the presence of the plates, and which results in the plates being pushed together. Electromagnetic radiation can be pictured as waves flowing through space at the speed of light. The waves are not waves of anything substantive, but are ripples in a state of a theoretically defined field. However these waves do carry energy (and momentum), and each wave has a specific direction, frequency and polarization state. Each wave represents a "propagating mode of the electromagnetic field." Each mode is equivalent to a harmonic oscillator and is thus subject to the Heisenberg uncertainty principle. From this line of reasoning, quantum physics predicts that all of space must be filled with electromagnetic zero-point fluctuations (also called the zero-point field) creating a universal sea of zero-point energy. Thus, it is clear from the explanation that our universe appeared from something instead of nothing assumed by Einstein. According to my explanation in the previous articles, that means from Big-Rip singularity to 11-dimensional space-time the universe remained in the stage which may compared with the state like gases. The density of this energy depends critically on where in frequency the zero-point fluctuations cease. Since space itself is thought to break up into a kind of quantum foam at a tiny distance scale called the Planck scale $\left(\mathbf{1 0}^{-33} \mathbf{~ c m}\right)$, it is argued that the zero point fluctuations must cease at a corresponding Planck frequency $\left(10^{43} \mathbf{H z}\right)$. If that is the case, the zero-point energy density would be 110 orders of magnitude greater than the radiant energy at the centre of the Sun).

Zero-point energy has the desired property of driving an accelerated expansion, and thus having the requisite properties of dark energy, but to an absurdly greater degree than required, i.e. 120 orders of magnitude. Work by Christian Beck, and Michael Mackey, 2006 may have resolved the 120 order of magnitude problem. In that case dark energy is nothing other than zero-point energy. In "Measure-ability of vacuum fluctuations and dark energy" and "Electromagnetic dark energy" they propose that a phase transition occurs so that zero-point photons below a frequency of about $1.7 \mathrm{THz}$ are gravitationally active whereas above that they are not. If this is the case, then the dark energy problem is solved: dark energy is the low frequency gravitationally active component of zero-point energy. SED studies published in the 1990s showed that a mass-less point-charge oscillator accelerating through the zero-point field will experience a Lorentz force (from the magnetic components of the zero-point fluctuations) that turns out to be directly proportional to acceleration, allowing the derivation of the fundamental $\mathrm{F}=$ ma relationship of mechanics from electrodynamics. That points to the electromagnetic quantum vacuum as the origin of forces which appear as inertial mass. It was said by Andrei Linde, 2005 that our knowledge of the world begins not with matter but with perceptions. It is possible that consciousness, like space-time has its own degrees of freedom and that neglecting it leads to a description of the universe that is fundamentally incomplete. He finally stated: all matter in the universe was produced with quantum fluctuations after the end of inflation. Quantum effects combined with inflation made the universe not only infinitely large but also immortal and afforded a beautiful and self-consistent pattern.

\section{Actual Source Of Information Field}

Excellent articles of Amit Goswami, 2005. "Morphogenesis", the growth of a complete organism from a single cell embryo, is a phenomenon of biological order of unprecedented precision timing of correlated events of both spatial and temporal nature. One doesn't see anything like this in dealing with inanimate matter. Naturally, beginning with Erwin Schrodinger, 1959, 1986, an impressive group of scientists have suggested that the currently known laws of physics may not be enough to explain morphogenesis. Many biologists have propounded the idea of a morphogenetic field -- a condition of space that retains the memory of the form that the embryo evolves towards under the guidance of the fields. Sheldrake, 2005 proposed morphogenesis has overtones of teleology to many. One has recently injected new principles in the old idea of morphogenetic fields to incorporate teleology, non-locality, and downward causation. Sheldrake's morphogenetic fields are purposive and non-local. They are not material as we understand. They are capable of downward causation in matter 
through a new principle called "morphic resonance". As soon as a new form comes about, it sets up its own field which is continually reinforced with its ongoing replication, thus explaining the memory exhibited in morphogenesis. However, there is the fundamental question of the source of the morphogenetic fields that resonate with matter, since this exhibits an implicit dualism. Morphogenesis can be given sound footing on the basis of Quantum principles applied in the conceptualization of the living cell within the context of a new science -- science within consciousness, a science based on the primacy of consciousness. This will take up the necessity of a new formulation of biology within the primacy of consciousness and indicate how morphogenesis theory is a precursor and a special case of this new theory. The question of experimental verification is necessarily required. In this circumstances we introduce a generalise conception about the series of new energy sources SU(6), SU(12)...etc. We consider that our present universe was appeared by a long process of the symmetry breaking from infinite space-time, mathematically it was explained by the theory of Gaussian transformation law of energy groups. After the symmetry breaking of SU(11), the 10-dimensional space-time created the first stage of dark matter phases whose realisation as the consciousness and which is controlling the whole universe of the second stage that means our physical universe or so called 4-dimensional universe and creating a pseudo-electromagnetic interaction field producing a neutral current flow which affected everything of our universe including biological particles and hence created a "morphic resonance". Therefore, morphogenesis is non-local. It is very interesting that within the standard model of physics we get a "weak neutral current" by one neutral particle of SU(2) of zero mass and charge by interaction with U(1). Similarly we found another strong neutral current by the interaction of SU(6) with U(1). Experimentally, European research centre "CERN" confirmed that there exist a "Flavour changing neutral current". So, it may be compared with a magnet that if we cut the magnet that generates magnetic field, we get two of the same type magnetic fields--that means two halves of the original one.

Indeed, with these properties of morphic fields, (a) downward causation and purposiveness, (b) nonlocality and (c) morphic resonance with memory property all of the difficulties of biological morphogenesis of materialist theories are resolved. But morphic field theories, seemingly has an implicit dualism, if postulated to transcend matter. They are capable of influencing matter. Resonance phenomenon in radio needs electromagnetic wave propagation between a source and a receptor. In this theory, there is the receptor (the living object) and there is the information being received (the morphogenetic fields). Here we consider the source of morphogenetic information is due to the latent (or pseudo) energy group SU(6) of the first stage, which creates some inherent characters in nature and mind of the living things called intelligences. This intelligence expressed by consciousness itself, looked upon as the ground of all being. Consciousness acts on matter in conjunction with a vital body that contains the blueprints of biological forms. That is the source of morphic information. And the function of morphic fields and resonance is carried out by consciousness simultaneously choosing actuality from possibility forms of both the vital and the physical body of an organism. And yet, this process does not involve dualism. Instead, the process involves self-referential quantum measurement. In science within consciousness, we propose that there is a creative programmer--consciousness itself. Consciousness is creative and changes the contexts of learning in the programs of the living cell. Not only is morphogenesis explainable with this hypothesis but also is the evolution of new forms. And not only morphogenesis and evolution, but the door is also opened to understand Life itself as a manifestation of consciousness as different from insentient matter. Such type of consciousness may arise from the Gaussian energy groups SU(12), SU(24),...etc., involving with the infinite number of extra-dimensions other than the energy group SU(11) of the 10-dimensional space-times . For Life, there has to be a new organizing principle. But it is unlikely that such organizing principles reside in matter emerging at the appropriate level of complexity of the universe and the planets etc. where the situations of biological temperatures are available.

There is one more important question --the question of meaning. Computers process symbols but not the meaning of symbols. The meaning exists in the mind of the programmer. Computer scientists routinely assume such a psychophysical parallelism in their theories as discussed by Varela, Thomson, and Rosch, 1991, and even by Dennett 2007. Similarly, the meaning of living functions and attributes such as maintenance, reproduction, evolution, and form must be carried in a separate body within consciousness. This body is traditionally called the "vital body. In summary, we need a theory of bio-physical parallelism --a simultaneous and parallel functioning of a vital body and a physical body. But the introduction of the vital body (including consciousness) in biology surely raises the spectre of dualism. It is the solution of the problem of dualism with the help of quantum measurement theory that makes science within consciousness a viable concept for biology. If there are two separate dual bodies, in two phases, what mediates their interaction and without violating conservation laws such as the Conservation laws of Energy? - is the latent energy group SU(6) which mediates the parallelism between the physical and the vital body by creating an electromagnetic wave propagations. And maintains the exact correspondence, if the vital body exists in a parallel existence with the physical in exact correspondence (biophysical parallelism as enunciated by Leibnitz), the problem of dualism still larks.Evidence for the quantum nature of the vital body is found in the demonstration of non-locality. Qigong 
masters can non-locally affect the vital field of plants enough to enhance or reduce the rate of biochemical reactions involved in plant growth. Further note that the quantum picture enables us to think about the physical and the vital worlds differently from what we are used to. Normally, we tend to think of both of these worlds as made of substances (something "concrete"). Sure, the vital substance is more subtle. We cannot quantify it in the same way as we can the physical. But it is still a substance, or so we think. We must change our view. Even the physical is not substance in the ordinary sense, let alone the "vital". Both physical and vital worlds remain as quantum superposition of possibilities until consciousness gives them substantiality by collapsing an actual experience. The quantum system in the cell along with Macro-measurement apparatuses that amplify its states (and also make a record of the actualized states upon measurement) --whose states are collapsed in a correlated parallel fashion with the states of the vital body -- can be said to have mapped the blueprint, the meaning, contained in the latter. This is similar to how we write software on computer hardware. The maps are inherited by all the cells of the body of a multi-cellular organism through cell division. The shared maps can solve some of the profound puzzles of biological morphogenesis.

\section{Conclusion}

Quantum physics indicates that consciousness is related to the awareness that an electron appears to show in the wave/particle duality (double slit experiment). Quantum physicists have shown that the electron behaves differently when being observed by a human. When the electron is not being observed, the electron behaves like a wave, but when an observing instrument is placed in the experiment, the electron behaves like a particle. This experience indicates that the electron will change its behaviour/reality depending on whether or not the electron is being observed as if the electron is aware that it is being observed. This awareness is very similar, if not the same, as human awareness and may be related to the same consciousness. Thus consciousness understood if there creates lives otherwise it becomes pseudo but working silently for the formation of our universes and then behaves like entanglement. Consciousness is, therefore, a non-material entity capable of independent, eternal existence, and not a property but may be used as property. Consciousness is not emergent, and is eternal similar to the electron. It can remain localized in the human brain and interact with the brain, and thereby, control the activities of the human body. While electrons in the brain behave as particles, these electrons prevent the consciousness from realizing that it is part of a larger whole. When the electrons behave as a wave, the consciousness becomes aware of its existence outside the human mind, which makes OBE and NDE possible. Whenever the electron wave function collapses, the OBE and NDE ends and the person returns to their physical body and its perception of reality similar to the collapsing of the wave function in the double slit experiment in quantum physics. During the OBE and NDE while the electron is behaving as a wave function, consciousness can leave the brain and go into an independent floating existence outside the human body where it can travel independent of space-time similar to the entangled electron.

It has been shown using such techniques as PET and MRI that the above process of receiving data from a stimulus by a sensory organ, transmitting them to the brain, computing and processing the data, and passing the processed data to consciousness, can be reversed by hypnotizing a person. When hypnotherapies suggests, for example, that he/she is seeing red light to a hypnotized subject, all above processes take place in the brain as if the subject is actually seeing red light. The consciousness gives the brain a perception of reality that is different from the hypnotherapies' perception of reality similarly to an observer in the example of general relativity where one observer on Earth watches another travel away at the speed of light. To the travelling observer, his perception of reality is different than the observer watching from Earth, and as a result, his experience of time is different than the observer on Earth.

The ability of a person to describe what his/her consciousness had observed or heard while it is in a des-embodied state makes us to believe that memory is, at least partly, non-material and unaffected by collapsing the wave function. Quantum systems are essentially unified, and so are our thought processes. David Bohm says, "Thought processes and quantum systems are analogous in that they cannot be analysed too much in terms of distinct elements because the intrinsic nature of each element is not a property existing separately from and independently of other elements, but is instead a property that arises partially from its relation with other elements."We can conclude that consciousness is a quantum mechanical entity that can have an independent existence but related with the matter substances as it explained that the bosons of the Gaussian energy group $\mathrm{SU}(6)$ changes to the bosons of $\mathrm{SU}(5)$ or vice-versa. It can localize in the human brain when the electron is in a particle state. This provides the necessary quantum mechanical base conductive for it to interact with and function in the brain. When the state changes to that of a wave, consciousness takes flight and starts floating. It takes away with it at least a part of the contents of the memory. It possesses the ability to acquire visual, auditory and olfactory information in spite of the fact that there are no sense organs associated with it. This information is produced by the consciousness projection of a different reality caused by the change in state of the electron, which one may interpret later as a dream or hallucination that comes from an altered perception of reality. 
The major stumbling block in solving the brain-mind problem is how does the brain-mind bind together millions of dissimilar neuron activities into an experience of a perceptual whole. How does the "I" or "self" or the perceived wholeness of one's world emerge from a system consisting of so many parts, billions of neurons. What creates the "Oneness" of thought processes? What creates individuality and "I"-ness or "self"? What creates feelings, free will, and creativity? The problem is solved only by making a complete total body assumed like an atom/molecule with the combination of two different characters of electro-magnetic wave functions producing in two different phases by the symmetry breaking of SU(11) \& SU(5) and then formation of biological particles, otherwise does not create such feelings etc.

In classical science, the most ordered structure that we can find is the crystal. Crystals are rigid, immovable structures. In Bose-Einstein condensates, the quantum properties allow both a "fluid" order and a high degree of unity. Each particle in a Bose-Einstein condensate produces and also fills all the spaces by the strong force SU(6) and all the time in whatever container that holds the condensate. Many of their characteristics are correlated. They behave holistically as one. The condensate acts as one single particle. There is no "noise" or interference between separate parts. This is why super fluids and super conductors have their special frictionless qualities and lasers become so coherent. Super conductors, super fluids, and lasers are BoseEinstein condensates. The photons of a laser beam overlap their boundaries and behave as one single photon and the whole system can be described by a single equation. Hence, the part always includes the whole like in fractal geometry. Super conductors, super fluids, and lasers are either very low temperature or very high energy systems. Super conductors and super fluids lose their quantum coherence long before they reach room temperature. Quantum coherence at body temperature in body cells was found by Herbert Frohlich. Prior to that, quantum physicist Fritz Popp discovered that biological tissue emits a weak glow when stimulated at the right energy levels. Cell walls of biological tissue contain countless proteins and fat molecules which are electrical dipoles. When a cell is at rest, these dipoles are out of phase and arrange themselves in a haphazard way. But when they are stimulated they begin to oscillate or jiggle intensely and broadcast a tiny microwave signal. Frolich found that when the energy flowing through the cell reaches a certain critical level, all the cell wall molecular dipoles line up and come into phase. They oscillate in unison as though they are suddenly coordinated. This emergent quantum field is a Bose-Einstein condensate and has holistic properties common to any quantum field. Consciousness may work in a similar matter.

Zohar, 1990 suggests that ion channel oscillations in neurons are quantum phenomena; which, as in Frolich systems, generates a coherent quantum electric field. It is a Bose-Einstein condensate. Existence of such large scale coherent electrical fields across the brain explains how a large number of disparate and distant neurons can integrate their information to produce a holistic picture, hologram. The quantum entanglement proof that has been developed fairly recently says that non-local [instantaneous or faster than light that means we consider photons of SU(6)] quantum correlations exists between particles apparently separated in space and time has helped scientists to understand these effects, quantum entanglement, also called the quantum non-local connection, is a property of a quantum mechanical state of a system of two or more objects in which the quantum states of the constituting objects are linked together so that one object can no longer be adequately described without full mention of its counterpart - even if the individual objects are spatially separated in a space-like manner.

The crucial distinguishing feature of Bose-Einstein condensate is that many parts which go to make up the ordered system not only behave as a whole, but they become whole. Their identities merge and overlap in such a way that they lose their individuality entirely. This is a quantum property. Such a large quantum synchronicity exists in and accounts for the special properties of lasers, super conductors, and super fluids. Only this type of quantum correlated condensed state could explain the unbroken wholeness of the thought process. Consciousness behaves as a fractal. This is similar to a hologram where small fractal parts are identical to the whole part. The property of the brain which is the non-local quantum correlate or the Bose-Einstein condensate behaves as above. It creates a unity from the diverse bits of information drawing them to a meaningful whole. The millions of sensory data from sense organs received every moment are channelled to various disparate areas of the brain and processed by the computing facility of the brain. Consciousness receives this processed information and creates a holistic scene, hologram. It is this integration of all the processed bits of information to create a one whole that creates the identity as a person, the self or the I-ness. Here is the coherent non-local quantum correlation of the brain and it is an emergent property. Consciousness is a non-material entity in the quantum domain that is capable of independent existence. Consciousness can remain localized in the brain so long as the emergent quantum particle state does not change, just as an electron which is a quantum entity can remain localized in an atom so long as the energy of the electron matches the quantum state it occupies. Whenever the property breaks down, the mathematical function that governs the behaviour of quantum particles changes, i.e. the electrons changes its behaviour from that of a particle to a wave function. When this happens in the brain, consciousness can leave the brain and take up a floating existence in the way an electron leaves its atom if it acquires excess energy and starts a floating existence as a free electron. Consciousness can return to 
the brain if the property is re-established and the wave function collapses and the particle function prevails. This model may explain all the observed properties of human consciousness including Psi-phenomena, NDE, OBE and reincarnation. Thus we required balance energy for living otherwise death. Since all information transfer in a non-local quantum correlation is instantaneous, just like the speed of an electron and light is instantaneous, it explains the speed of human action. It can be extended to explain phenomena such as telepathy and also explains the individual identity, or the I-ness or self. It is initially puzzling that the same type of experiments that show a correlation between consciousness and individual neuron also show a correlation between the global gamma synchrony and consciousness. So which of these produces consciousness, the individual neurons or the gamma synchrony? Recent studies suggest that activity in individual neurons correlates with the gamma synchrony when a number of the neuron's neighbours were also active. This agrees with studies showing 'hot spots' of activity in the brain also correlated with consciousness. Here we are perhaps left with the concept that the brain is a gate to the fundamental level of the universe, in the literal sense of a mechanism that allows or prevents entry. All of this may seem very speculative, but against this has to be set the lack of explanation in classical physics for the 'something it is like' or the ability to have choice or preference that we find in consciousness. We need all the explanation by quantum physics which shows the appropriate way for solutions.

\section{Acknowledgement}

The author is thankful to Prof. Subenoy Chakraborty, Department of Mathematics, Jadavpur University, Kolkata, West Bengal, India, for the discussion about the universe and my friend Dr. Swapan Raha, Department of Mathematics, Viswabharati, Santiniketan, West Bengal, India.

\section{References}

[1]. P. J. E. Peebles and B.Ratra, Astrophys. J. Lett . 325. L17 (1988): and R. G. Vishwakarma, Class. Quant. Grav. 14. 945(1997). F. Hoyle. G. Burbidge and J. V. Narlikar, Mon. R. Astron. Soc. 286.173(1997). T. A. Appelquist, A. Chodos and P. G. O. Freund, Modern Kaluza-Klein Theories, Frontier s in Physics Series, (Volume 65), 1986 (E d. Addison Wesely). Brian Greene, 'The Elegant Universe', W. W. Norton \&Company, New York (1999), pp. 357-358. G. Riess et al. Astrophys. J. 560, 49(2001).Everett, H. (1957).Relative state formulation of quantum mechanics.

[2]. In Quantum Theory and Measurement, Wheeler, J. A., and zurek, W. H. (eds), Princeton University Press, 1983; Originally in Reviews of Modern Physics, 29, 454. Engel, G.S., Calhoun, T.R. , Read, E.L., Ahn, T-K., Mancal, T. , Cheng, Y-C. , Blankenship, R.E., Fleming, G.R., (2007). Evidence for wave-like energy transfer through quantum coherence in photo synthetic systems, Nature 446: 782. Bhadra N.K. (2012): The complex Model of the Universe, IOSR-JM, ISSN: 2278-5728, vol.2, 4, pp-20; and The complex model of the quantum universe, ISSN: 2278-5728.IOSR Journal of Mathematics (IOSR-JM) vol.4, Issue. 1(Nov.-Dec2012),pp-20. "The Complex Quantum-State of Black-Hole and Thermo-statistics" (IOSR-JM: e-ISSN: 2278-3008, p-ISSN: 2319-7676. Volume 8, Issue 5 (Nov. -Dec. 2013), PP 01-19)].Bhadra NK; THE COMPLEX QUANTUM AND CLASSICAL PSEUDO-TACHYONIC UNIVERSE; (IOSR-JM) e-ISSN: 2278-5728,p-ISSN: 2319-765X, Volume 8, Issue 3 (Sep. -Oct. 2013), PP 15Einstein, A. de-Sitter, W. (1932): On the relation between the expansion and mean density of the universe. Proc. Natl. Acad. Sci.,(USA), 18,213. Hawking S.W(1984): The quantum state of the universe, Nucl. Phys. B239.257.Davies, P.C.W. (1974): The Physics of Time Asymmetry (Surrey University Press/ University of California Press) -1976a Proc. R. Soc. A. 351 139; -1976b Nature 263 377; -1977a Proc. R. Soc. A. 353 499; -1977b Space and Time in the Modern University Cambridge: Cambridge University Press).

[3]. Bandyopadhyaya, A (2011) Direct experimental evidence for quantum states in microtubules and topological invariance. Towards a science of consciousness 2011, Sockhalm, Sweden, HYPERLINK,, http://www.consciousness.arizona.edu. " Raggett S. Quantum Mind. Exploring Quantum Theories of Consciousness http://quantum-mind.co.uk/ Meijer D K F and Korf J (2013). Quantum modeling of the mental state: the concept of a cyclic mental workspace. Syntropy Journal, (1), pp 1-41 http://www.lifeenergyscience.it/english/2013-eng-1-1.pdf Meijer D K F (2014). The Extended Brain: Cyclic Information Flow, in a Quantum Physical Realm. Neuro Quantology, vol. 12, pp 180-200. Abstract: http://www.neuroquantology.com/index.php/journal/article/view/754 Goswami, A (2003). A QuantumExplanationofSheldrake'sMorphicResonance.http://www.stealthskater.com/Documents/Consciousness_32. pdf Bromberg F (2000).On Goswami's monistic idealism worldview. http://www.cs.iastate.edu/ bromberg/BrombergPhyl465Final Paper. pdf Hameroff S, Penrose R (2013). Consciousness in the universe. A review of the 'Orch OR' theory. Phys Life Rev., http://dx.doi.org/10.1016/j. plrev.2013.08.002 King CC (2012). The Cosmology of Conscious Mental States. Genotype 1.0.0 21-5-13 - 14-8-13 Abbott D [and others] (2008). Quantum aspects of life, London: Imperial College Press. Aharonov Y, Popescu S, Tollaksen J, (2010). A time-symmetric formulation of quantum mechanics, Physics Today, November, 27-32. Allefeld C, Atmansacher H, Wackerkanm J, (2009). Mental states as macrostates emerging from brain electrical dynamics. Chaos; 19 (1) 1-32. Amoroso R L (1999).

[4]. An Introduction to Noetic Field Theory: The Quantization of Mind. Noetic Journal Vol.2 No.1, 28-39 Arndt M, Juffmann T and Vedral V, (2009). Quantum physics meets biology, HFSP Journal. Aspect A, Grangier P and Roger G, (1982). Experimental realization of Einstein-Podolsky-Rosen-Bohm Gedankenexperiment: a new violation of Bell's inequalities. Phys. Rev. Lett. 48: 91-94. Atmanspacher, H, Filk T, Römer H, (2004). Quantum Zeno features of bi-stable perception. Biol. Cybern. 90: 33-40. Atmanspacher H, (2007). Contextual emergence from physics to cognitive neuroscience. Journal of Consciousness Studies; 14 (1/2):18-36. Atmanspacher H, (2011). Quantum Approaches to Consciousness, The Stanford Encyclopedia of Philosophy (Summer 2011 Edition), Edward N. Zalta (ed.), Atmanspacher H, Primas H, (1977). The Hidden Side of Wolfgang Pauli: An eminent physicist's extraordinary encounter with depth psychology. Journal of Scientific Exploration; 11 (3): 369-386. Auletta G, Ellis GFR and Jaeger L, (2008). Top-down causation by information control: from a philosophical problem to a scientific research programme. J. R. Soc.

[5]. Interfacevol.5,no.27,11591172.http://plato.stanford.edu/archives/sum2011/entries/qtconsciousness/Bandyopadhya y A (2011). Direct experimental evidence for quantum states in microtubules and topological invariance. Abstracts: Toward a Science of Consciousness 2011, Stockholm, Sweden,

DOI: 10.9790/4861-0901025793 www.iosrjournals.org $\quad$ w Page


http://www.quantumbionet.org/admin/files/Massimo\%20Pregnolato\%20-\%20Rita\%20Pizzi\%202011.pdf Barrow J D and Tipler F J (1986). The Anthropic Cosmological Principle. Oxford University Press Baylor DA, Lamb TD, Yau KW, (1979). Responses of retinal rods to single photons. J Physiol. Mar; 288:613-34. Beck C and Mackey M, (2007). Measurability of vacuum fluctuations and dark energy. Physica A 379:101-110, arXiv:astro-ph/0605418v3 Beck F and Eccles J, (1992). Quantum aspects of brain activity and the role of consciousness. Proceedings of the National Academy of Sciences USA ; 89:11357-11361 Beck F and Eccles J, (2003). Quantum Processes in the Brain: A scientific basis of consciousness. In Osaka N (Ed.), Neural Basis of Consciousness. Amsterdam, Philadelphia: John Benjamins. Beck F, (2001).

[6]. Quantum brain dynamics and consciousness. In: The physical nature of consciousness. Ed. P. van Locke Amsterdam Benjamins; pp 83-116. Bekenstein J, (2003). Information in the holographic universe. Sci. Am. 289, 58-65. Bell J S, (1966). On the problem of hidden variables in quantum theory, Reviews of Modern Physics, 38, p. 447. Bergson H, Paul NM and Palmer WS, (1991). Matter and Memory, Zone Books. Berkovich S, (2001). On the "barcode" functionality of the DNA, or the phenomenon of Life in the physical Universe. http://arxiv.org/ftp/physics/papers/0111/0111093.pdf Bernroider G. Roy S. (2004). Quantum-Classical Correspondence in the Brain: Scaling, Action Distances and Predictability behind Neural Signals Forma, 19, 5568 Bernroider, G. \& Roy, S. (2005).

[7]. Quantum entanglement of $\mathrm{K}+$ ions, multiple channel states and the role of noise in the brain International Society for Optical Engineering (SPIE), vol. 5841 Bernroider, G. \& Summhamer, J. (2007). The role of quantum cooperativity in neural signalling .Quantum Mind 2007 Conference Abstracts Bernroider, G. (2003) - Quantum neurodynamics and the relationship to conscious experience - Neuroquantology, 2: 163-168 Blake T., Dotta B. T., Buckner C. A., Cameron D., Lafrenie R. M., Persinger M. A. (2011). Biophoton emissions from cell cultures: biochemical evidence for the plasma membrane as the primary source. Gen. Physiol. Biophys. 30, 301 Bode S, He AH, Soon CS, Trampel R, Turner R, et al. (2011). Tracking the Unconscious Generation of Free Decisions Using UItra-High Field fMRI. PLoS ONE 6(6): e21612. doi:10.1371/journal.pone.0021612 Bókkon I. (2009). Visual perception and imagery: a new hypothesis. Biosystems, 96, 178, 184.10.1016/j.biosystems.2009.01.005 Bókkon I., D'Angiulli A. (2009). Emergence and transmission of visual awareness through optical coding in the brain: a redox molecular hypothesis on visual mental imagery. Biosci. Hypotheses 2, 226- 232.10.1016/j.bihy.2009.01.009

[8]. Bókkon I., Dai J., Antal I. (2010a). Picture representation during REM dreams: a redox molecular hypothesis. Biosystems100, 79-86.10.1016/j.biosystems.2010.01.005 Bókkon I., Salari V., Tuszynski J., Antal I. (2010b). Estimation of the number of biophotons involved in the visual perception of a single-object image: biophoton intensity can be considerably higher inside cells than outside. J. Photochem. Photobiol. B Biol. 100, 160166.10.1016/j.jphotobiol.2010.06.001 Bohm D, (1990).

[9]. A new theory of the relationship of mind and matter. Philosophical Psychology.: 3: 271-286. Bohm D (1980). Wholeness and the implicate order, London: Routledge \& Kegan Paul Bohm D and Hiley B J (1987). An ontological basis for the quantum theory, Physics Reports, 144, pp. 323-348 Bohm, David \& B.J. Hiley, (1993): The undivided universe.

[10]. An ontological interpretation of quantum theory. London: Routledge Boly M, et al (2011). Preserved feed forward but impaired top-down processes in the vegetative state. Science 13 May 332 (6031): 858-862, DOI: 10.1126/science.1202043. Bouwmeester D et al. (1997). Experimental quantum teleportation. Nature, 390, pp. 575-579.Bromberg F.(2000 ).On Goswami's monistic idealism worldview. http://www.cs.iastate.edu/ bromberg/BrombergPhyl465FinalPaper.pdf Boyden, E. S, Zhang F, Bamberg E, Nagel G, Deisseroth K (2005). "Millisecond-time-scale, genetically targeted optical control of neural activity". Nat. Neurosci 8 (9): 1263-8. doi:10.1038/nn1525. PMID 16116447. Busemeyer J R and Bruza P D (2012).

[11]. Quantum models of cognition and decision. Cambridge University Press, Cambidge pp1-403. Carter P J (2014a). Quantum Spacetime and Consciousness, http://vixra.org/pdf/1312.0208v2.pdf Carter P J (2014 b). Imaginary Physics. http://vixra.org/pdf/1210.0124v3.pdf Chalmers D J. (2000). Facing Up to the Problem of Consciousness. http://consc.net/papers/facing.pdf Chaudhury S, Smith A, Anderson B, Ghose S, Jessen P (2009). Quantum signatures of chaos in a kicked top, Nature 461 768-771. Chin A.W., Prior J., Rosenbach R., Caycedo-Soler F.,. Huelga S.F,. Plenio M.B (2013). The role of non- equilibrium vibrational structures in electronic coherence and recoherence in pigment-protein complexes. Nat Phys, 9 pp. 113-118 Cho A. (2011). Furtive Approach Rolls Back the Limits of Quantum Uncertainty, Science 333 690-3. Clarke C J S (2001). Consciousness and non-hierarchical physics in: The physical nature of consciousness, Ed Philip van Looke, pub. Jon Benjamins Publishing, 2001, pp 191-217 Clarke, C J S (1995). The non-locality of mind', JCS 2 231-240 Clarke, C J S (2000).

[12]. Construction and reality: reflections on philosophy and spiritual/psychotic experience. in Psychosis and Spirituality: Exploring the New Frontier ed I Clarke, Whurr, London Clayton and Davies, P (2006).Re-emergence of Emergence, Oxford University Press Cramer J (1988). An Overview of the Transactional Interpretation. International Journal of Theoretical Physics 27, 227. Cramer J (2005) A Farewell to Copenhagen?, Analog, December. Davia CJ (2010) Life, Catalysis and Excitable Media: Dynamic Systems Approach to Metabolism and Cognition in: The Emerging Physics of Consciousness, (ed J.A. Tuszyn ski), Springer Berlin pp 255-292, Davies $\mathrm{P}$ and Gregersen N H (2010). Information and the Nature of Reality: From Physics to Metaphysics. Cambridge:Cambridge University Press. Davies P C W (2004). Quantum fluctuations and life. Available on: arXiv:quant-ph/0403017 Del Giudice, E. et al (2002). Effects of weak magnetic fields on ions. Bio-electromagnetics, 23, 522-530 Del Giudice, E., Doglia, S. Milani, M. \& Vitiello, G. (1988). Electromagnetic interactions in living matter.

[13]. In Fröhlich, H. ed. Biological Coherence and Response Dennett D (2007). Consciousness Explained. http://pages.uoregon.edu/donovan/writings/Chapter\%207\%20summary.pdf Deutsch D (1997). The Fabric of Reality, London: Allen Lane Di Biase F (2009a). A Holoinformational Model of Consciousness. Quantum Biosystems, 3, 207-220. Di Biase F (2009b). Quantum-holographic informational consciousness. NeuroQuantology, Vol 7 pp 657-664. Dixon, P. B., Starling, D. J., Jordan, A. N., \& Howell, J. C. (2009).

[14]. Ultrasensitive beam deflection measurement via interferometric weak value amplification. Physical review letters, 102(17),173601 Donald J A Evidence supporting information processing in animals -, with references to David Deutch, T. Kanade and others. http://w2.eff.org/Net_culture/Consciousness/quantum_info_process.paper Dotta B. T., Persinger M. A. (2011). Increased photon emissions from the right but not the left hemisphere while imagining white light in the dark: The potential connection between consciousness and cerebral light. JCER2, 1463-1473. 
Dotta B. T., Saroka K. S., Persinger M. A. (2012). Increased photon emission from the head while imagining light in the dark is correlated with changes in electroencephalographic power: Support for Bókkon's Biophoton Hypothesis. Neurosci. Lett. 513, 151-154.10.1016/j.neulet.2012.02.021

[15]. Duncan W L( 2010). The Quantum Universe: An Information Systems Perspective. http://criticalpath.itgo.com/Articlesanscover.html Dyer, M.G. (1994). Quantum physics and consciousness, creativity, computers: A commentary on Goswami's quantum-based theory of consciousness and free will. The Journal of Mind and Behavior, 15, 265-290. Edelman GM, Tononi G (2000). The universe of consciousness. How matter becomes imagination. Basic Books New York Edwards J. (2005). Is consciousness only a property of individual cells? J Consciousness Studies; 12: 429-457. Edwards J, (2008). Are our spaces made of words? Journal of Consciousness Studies, 15, No. 1, , pp. 63-83 Edwards P (1967). Panpsychism, in: Paul Edwards (ed.) The Encyclopedia of Philosophy, vol. 5. Ellis G F R (2005). Physics and the real world. http://www.mth.uct.ac.za/ ellis/realworld.pdf Emerson Daniel J, Weiser BP, J, Z. Liao, O Taratula, A Fiamengo, X. Wang, K Sugasawa, Smith A B., III, Eckenhoff R G, and D Mochowski (2013).

[16]. Direct Modulation of Microtubule Stability Contributes to Anthracene General Anesthesia. J Am Chem Soc. Apr 10, 2013; 135(14): 5389-5398 doi: 10.1021/ja311171u Engel GS, Calhoun TR, Read EL, Ahn TK, Mancal T, Cheng YC, Blankenship RE, Fleming GR (2007). Evidence for wavelike energy transfer through quantum coherence in photosynthetic systems. Nature, 446: 782-786. Feferman, S (1996). Penrose's G"odelian argument. http://math.stanford.edu/ feferman/papers/penrose.pdf Flanagan BJ (2007). On the unification of Mind and Matter. NeuroQuantology, Vol 5, Issue 4, Page 331-345 Freeman, W J, and Vitiello G (2008). Dissipation and spontaneous symmetry breaking in brain dynamics. Journal of Physics A 41, 304042.Freeman, W J and Vitiello, G. (2006). "Nonlinear brain dynamics as macroscopic manifestation of underlying many-body field dynamics" Physics of Life Reviews 3(2), 93-118. Fröhlich H (1968). Long Range Coherence and Energy Storage in Biological Systems. Int. J. Quantum Chem; 2: 641-649.

[17]. Fröhlich, H (1970). Long-range coherence and the actions of enzymes .Nature, 228, 1093 Fröhlich, H (1975). Dielectric properties of biological materials. Neuron, 20, pp. 847-54 Fröhlich, H. (1985) Physics Letters, 110A 48 Gauger E, Rieper, E., Morton J.J.L., Benjamin S.C.,.Vedra V. (2011). Sustained quantum coherence and entanglement in the avian compass. Phys Rev Lett. 2011 Jan 28;106(4):040503. http://www.ncbi.nlm.nih.gov/pubmed/21405313 Geissler, H (1992). New magic numbers in mental activity. In: Eds. Geisler, H. et al - Cognition, Information Processing and Psychophysics Geissler, H. et al (1999). Ultraprecise quantal timing - Perception and Psychophysics, 6, pp. 707-26 Genovese M (2010). Interpretations of Quantum Mechanics and the measurement problem http://arxiv.org/abs/1002.0990v1 Georgiev, D. (2003). Electronic and magnetic fields inside neurons. http://cogprints.org/3190/1/solitons.pdf.

[18]. Georgiev, D. (2004). Bose-Einstein condensation of tunneling photons in the brain cortex as a mechanism of conscious action. http://cogprints.org/3539/1/tunnelling.pdf Germine M (2007). The Holographic Principle Theory of Mind. http://goertzel.org/dynapsyc/2007/holomind.htm Geroch, R., and Hartle, J.B. (1986) Computability and physical theories. Foundations of Physics 16:533. Gleiser M (2004), The three origins: cosmos, life, and mind, In: Science and ultimate reality, J.D. Barrow, P.C.W. Davies, and C.L. Harper (eds.), Cambridge: Cambridge University Press, pages 637-653. Globus, G (1995). Postmodern Brain. John Benjamins Goodman, E et al (1995). Effects of electromagnetic fields on molecules and cells. Int. Rev. Cytol., 158, pp. 279 - 339 Görnitz $\mathrm{T}(2012)$.

[19]. Quantum Theory as Universal Theory of Structures - Essentially from Cosmos to Consciousness, Advances in Quantum Theory, Prof. Ion Cotaescu (Ed.), ISBN: 978-953-51-0087-4,InTech, Available from:http://www.intechopen.com/books/advances-in-quantum-theory/quantum-theory-as universal theory-ofstructures-essential-from-cosmos-to-consciousness Goswami, A. (1989), The idealistic interpretation of quantum mechanics. Physics Essays, 2,385-400. Goswami, A. (1990). Consciousness in Quantum Physics and The MindBody Problem. The Journal of Mind and Behavior, winter 1990, 11, 1, p. 75-96. Goswami A. (1993). The Selfaware Universe. How consciousness creates the material world. New York: Tarcher/Putnam. Goswami, A (2003). A Quantum Explanation of Sheldrake's Morphic Resonance. http://www.stealthskater.com/Documents/Consciousness_32 .pdf Gray and Singer, (1989). Oscillatory responses in cat visual cortex exhibit inter-columnar synchronization which reflects global stimulus properties. Nature 338, 334 - 337 (23 March 1989); doi: 10.1038/338334a0 Greene B (2004). The Fabric of the Cosmos. About the Search for the Theory of Everything, The Spectrum, Utrecht.

[20]. Griffin D R (1997). Parapsychology, Philosophy, and Spirituality: A Postmodern Exploration, (SUNY Series in Constructive Postmodern Thought), State University of New York Press. Grof S, (1987). Beyond the Brain; Birth, Death and Transcendence in Psychotherapy, New York: State University of New York Press. Gurzadyan VG, Penrose R (2010). Concentric circles in WMAP data may provide evidence of violent pre-Big- Bang activity. arXiv:1011.3706 [astro-ph.CO]. Hagan, S., Hameroff, S.R., Tuszynski, J.A., (2002). Quantum computation in brain microtubules: Decoherence and biological feasibility. Phys. Rev. E. 65, 061901. Hagelin JS (1987). Is Consciousness the Unified Field? A Field Theorist's Perspective. http://www.mum.edu/pdf_msvs/v01/hagelin.pdf. Hameroff, SR, and Penrose, R (1996). "Conscious Events as Orchestrated Spacetime Selections", Journal of Consciousness Studies, 3(1): 36-53. Hameroff SR, (2007). The brain is both a neurocomputer and quantum computer. Cognitive Science; 31: 1035- 1045.

[21]. Hameroff S, Penrose R (2011) Consciousness in the Universe: Neuroscience, Quantum Space-Time Geometry and Orch OR Theory, In: Cosmology of Consciousness: Quantum Physics\& Neuroscience of Mind, pp 51- 102, Cosmology Science Publishers, Cambridge Hameroff S, Penrose R. (2013). Consciousness in the universe. A review of the 'Orch OR' theory Phys Life Rev http://dx.doi.org/10.1016/j.plrev.2013.08.002 Hamill OP, Marty A, Neher E, Sakmann B., Sigworth FJ (1981). Improved patch-clamp techniques for high resolution current recording from cells and cell-free membrane patches. Pflugers Arch, 391: 85-100 Hartmann L, Düer W and Briegel, HJ (2006). Steady state entanglement in open and noisy quantum systems at high temperature. Physical Review A 74 , 052304 Hawking S W and Mlodinov, L. (2010). The Grand Design. New York: Bantam Press. Hawking S. (1988). A Brief History of Time. Bantam Dell Publishing Group. Hayes, D. Griffith G.B,. Engel G.S (2013).

[22]. Engineering coherence among excited states in synthetic heterodimer systems . Science, 340 (6139) pp. 1431-1434 Heimburg, T, Jackson, AD (2005). On soliton propagation in biomembranes and nerves" Proc. Natl. Acad. Sci. U.S.A. 102 (2): 9790. h Henry RC (2005). The Mental Universe, Nature, 436(7): 29. Hildner R, Brinks, D, Nieder 
J.B, Cogdell R.J., van Hulst N.F. ( 2013). Quantum coherent energy transfer over varying pathw ays in single light harvesting complexes. Science, 340, 1448-51

[23]. Hiley BJ and Pylkkänen P (2005). Can Mind affect Matter via Active Information? Mind \& Matter, 3: 7 -27. Hiley, B. (2001). The Bohm Inerpretation and the Mind Matter Relationship. In Dubois, D. Ed. Computing Anticipatory Systems Hosten O. (2011). How to catch a wave, Nature 474 170-1. Hosten O., Kwiat P. (2008), Observation of the Spin Hall Effect of Light via Weak Measurements, Science 319 787-790. Holland, P. (1988)

[24]. Causal interpretation of Fermi fields. Phys. Lett., 128A, pp. 9-18 Holland P (1996). Quantum back-reaction and the particle law of motion. http://users.ox.ac.uk/ gree0579/index_files/back-reaction.pdf $\mathrm{Hu} \mathrm{H}$ and Wu M (2010). Current landscape and future direction of theoretical and experimental quantum brain/mind/consciousness research, J. Consc. Exploitation \& Research 1, 888-897. Hu H and Wu M (2004). Possible roles of neural electron spin networks in memory and consciousness. Cogprints ID 3544, see also NeuroQuantology, 2006: 5-16. Jahn RG and Dunne B J (2004). Sensors, Filters, and the Source of Reality. Journal of Scientific Exploration, 18(4): 547570. Jahn RG and Dunne B J (2007).

[25]. A modular model of mind/matter manifestations. Explore, 3:311-24, reprinted from J. Scientific. Exploration, 2001 Jensen CS, Rasmussen HB, Misonou H. (2011). Neuronal trafficking of voltage-gated potassium channels. Mol Cell Neurosci; 48: 288-297. Jiang, Y. MacKinnon, R. et al (2003). The principle of gating charge movement in a voltage-dependent $\mathrm{K}+$ channel, Nature, 423, pp. 42-8 Jiang, Y., MacKinnon, R. et al (2003) - X ray structure of a voltage-dependent K+ channel - Nature, 423, pp. 42-8 Jibu, M \& Yasue, K. (1992). A physical picture of Umezawa's quantum brain dynamics. In: Cybernetics and Systems Research, ed. R. Trappl World Scientific Jibu, M \& Yasue, K. (1993).

[26]. Introduction to quantum brain dynamics. In: Nature, Cognition and System ed, Carvallo, M. Kluwer Academic Jibu, M. et al (1997). Evanescent photon and cellular vision. Biosystems, Vol. 42, Issue 1, March 1997, Pages 65-73. http://www.sciencedirect.com/science/article/pii/S0303264797016869

[27]. Jibu, M., Hagan, S., Hameroff, S. Pribram, K. \& Yasue, K. (1994). Quantum coherence in microtubules Biosystems 32, 195-209 Jung, CG and Pauli W (1955). The Interpretation of Nature and the Psyche. New York, Bollinger. Kafatos M (2009). Cosmos and Quantum: Frontiers from the Future. J. Cosmol. Vol. 3, pp $511-528$ Kafatos, M, Tanzi R E, and Chopra D (2011).

[28]. How Consciousness Becomes the Physical Universe. Journal of Cosmology, Vol. 14, pp 1-11 Kaivarainen A. (2005). Hierarchic model of consciousness. NeuroQuantology, Issue 3, 180-219 Kak S (2009). The Universe, Quantum Physics and Consciousness. J.Cosmol. Vol.3, pp 500-510 Kauffman SA (2008). Reinventing the Sacred: A New View of Science, Reason, and Religion. New York: Basic Books. Kauffman SA (2012). Is there a "”poised realm between quantum and classical worlds? http://stuartkauffman.com/index_25 1612352741.pdf Khrennikov, A. (1999) . Classical and quantum mechanics on information spaces. Found. Phys., 29, pp. 1065- 98 Khrennikov, A. (2002) . On cognitive experiments to test quantum-like behaviour of mind. Rep. Växjö University: Math. Nat. Sc. Tech., N7

[29]. Khrennikov, A. (2003). Quantum-like formalism for cognitive measurements . Biosystems, 70, pp. 211-33 Khrennikov, A. (2004) . Information dynamics in cognitive etc. phenomena. Fundamental Theories of Physics: Berlin-Heidelberg: Springer Khrennikov A. (2006). Quantum-like brain . BioSystems, 84 (3), pp. 225-41 King CC. (1989). Dual-Time Supercausality, Physics Essays, 2(2): 128-151. King CC. (2003). Chaos, Quantum-transactions and Consciousness, NeuroQuantology, 1(1): 129-162. King CC. (2011). The Central Enigma of Consciousness, Nature Precedings, 2008 JCER. King CC. (2012).

[30]. The Cosmology of Conscious Mental States. Genotype 1.0.0 21-5-13 - 14-8-13King CC. (2014). Space, Time and Consciousness. http://www.dhushara.com/stc/ct.htm King U (1996). Spirit of Fire: The Life and Vision of Teilhard de Chardin. Maryknoll, NY: Orbis. Knopf Doubleday Publishing Group (Random House). Koch C and Hepp K (2006). Concept Quantum mechanics in the brain. Nature 440, 611 (30 March ), doi:10.1038/440611a Kocsis, S., Braverman, B., Ravets, S., Stevens, M. J., Mirin, R. P., Shalm, L. K., \& Steinberg, A. M. (2011). Observing the average trajectories of single photons in a two-slit interferometer. Science,332(6034), 1170-1173. Kumar M (2009). Quantum. Einstein, Bohr and the Great Debate about the Physics, Amsterdam: Macmillan. Kurzweil R. (2005). The Singularity is Near. When Humans Transcend Biology. Viking. László E (2004). Cosmic vision.

[31]. The dawn of the integral theory of everything. Rochester, Inner Traditions International László, E. (2007).The Akashic Field. New York: Dutton Lehmann D., Strik W. K., Henggeler B., Koenig T., Koukkou M. (1998).

[32]. Brain electric microstates and momentary conscious mind states as building blocks of spontaneous thinking: I. Visual imagery and abstract thoughts. Int. J. Psychophysiol. 291-11. Li, J and Paraoanu, GS (2009). Generation and propagation of entanglement in driven coupled-qubit systems. New Journal of Physics 11, 113020. Lima, S Q and Miesenböck G (2005). Remote control of behavior through genetically targeted photostimulation of neurons. Cell 121 (1): 141-52. doi:10.1016/j.cell.2005.02.004. PMID 15820685 Libet B. (2006). Reflections on the interaction of the mind and brain. Prog Neurobiol. 78(3-5): 322-326. Life. Perseus Press: Boston, MA Linde A (2003).

[33]. Inflation, Quantum Cosmology, and the Anthropic Principle, in Science and Ultimate Reality: From Quantum to Cosmos, honoring John Wheeler's 90th birthday. Barrow JD, Davies PCW and Harper CL eds. Cambridge University Press. Lloyd S (2011). Quantum coherence in biological systems. J. Phys.: Conf. Ser. 302, 012037 Journal of Physics: Conference Series 302 (2011) 012037 doi:10.1088/1742-6596/302/1/012037 Lockwood, M. (1989). Mind, Brain \& the Quantum - Blackwell ISBN 0-631-18031-1 Lundeen J. S., Steinberg A. M. (2009). Experimental Joint Weak Measurement on a Photon Pair as a Probe of Hardy's Paradox. Physical review letters, 102 020404. Lundeen J.S., Sutherland B., Patel A., Stewart C, Bamber C. (2011). Direct measurement of the quantum wave function, Nature 474 188-191. Luo, L. Lu J (2011).

[34]. Temperature dependence of protein folding deduced from quantum transition http://arxiv.org/abs/1102.3748 Mandelbrot BB (1977). The Fractal Geometry of Nature. New York: Freeman. Marcer PJ and Schempp W (1997). Model of the Neuron Working by Quantum Holography, Informatica 21: 519-534 Marshall I (1989). Consciousness and Bose-Einstein condensates New Ideas in Psychology, 7: 73-83. Martin F, Carminati F, Carminati G G (2013).Quantum information theory to unconscious and consciousness. NeuroQuantology, 12: 16 33 Matsuno K (1999). Cell motility as an entangled quantum coherence. BioSystems 51, 15-19. Matsuno K. and Paton RC (2000). Is there a biology of quantum information? BioSystems 55: 39-46. McFadden J (2001). 
Quantum Biology. Norton, New York McFadden J and Al-Khali (2001). A quantum mechanical model of adaptive mutation. Biosystems 50, 203-211. http://www.surrey.ac.uk/qe/pdfs/mcfadden_and_al-khalili.pdf

[35]. McGinn C (1999). The Mysterious Flame: Conscious Minds in a Material World, New York: Basic Books. Meijer D K F and Korf J (2013)

[36]. Quantum modeling of the mental state: the concept of a cyclic mental workspace. Syntropy Journal, (1), pp1-41 Meijer D. K. F (2012). The Information Universe, on the missing link in concepts on the architecture of reality. Syntropy Journal,(1), pp 1-64 Meijer D. K. F (2013b). Immortality: Myth or becoming Reality ? Syntropy Journal; (3), pp 166-203. http://www.lifeenergyscience.it/english/2013-eng-3-04. pdf Meijer D K F. Information: what do you mean ? Syntropy Journal, 2013 a; (3), pp 1-49. http://www.lifeenergyscience.it/english/2013-eng-301.pdf Meijer D. K. F. (2014). The Extended Brain: Cyclic Information Flow, in a Quantum Physical Realm. Neuro Quantology, vol. 12, pp 180-200.Abstract: http://www.neuroquantology. com/index. php/journal/article/view/754 Melkikh A V 2013).

[37]. Biological complexity, quantum coherent states and the problem of efficient transmission of information inside a cell, Bio-Systems; 111: 190-8 Melkikh A V (2013). Quantum field theory solves the problem of the collapse of the wave function; http://arxiv.org/abs/1311.0205 Menini A., Picco C., Firestain S. (1995).Quantal-like current fluctuations induced by odorants in olfactory receptor cells. Nature 373, 435 - 437 (02 February); doi: 10.1038/373435a0 Merali Z. (2010). Back From the Future, Discover Magazine August 26 Minkowski H (1952). In: Lorentz HA, Einstein A, Minkowski H and Weyl H, (1952). The Principle of Relativity: A Collection of Original Memoirs on the Special and General theory of Relativity. New York: Dover Mitchell ED and Staretz R (2011).The quantum hologram and the nature of consciousness; Journal of Cosmology14:1-35. Murphy N (2011).

[38]. Avoiding Neurobiological Reductionism: the role of downward causation in complex systems, in Moral Behavior and Free Will. A Neurological and Philosophical Approach, eds Juan José Sanguineti, Ariberto Acerbi, José Angel Lombo Murphy, N. and W.R. Stoeger, SJ. (eds.). (2007). Evolution and Emergence: Systems, Organisms, Persons, Oxford: Oxford University Press. Nagel T (2012).

[39]. Levels of Complexity and Levels of Reality", in The Emergence of Complexity in Mathematics, Physics; Chemistry, and Biology, Proceedings of the Plenary Session of thePontifical28AcademyofSciences,2731October 1992.Seealsoin2011:http://bdigital.ufp.pt/bitstream/10284/2392/3 1119-130.pdf Ouyang, M, Awschalom D D (2003): Coherent Spin Transfer Between Molecularly Bridged Quantum Dots. Science, Vol. 301 no. 5636, pp. 1074-1078. DOI: 10.1126/science.1086963 Pauli W, (1994): Writings on Physics and Philosophy, ed. by C.P. Enz and K. von Meyenn. Springer, Berlin. Penrose R (2004). Twistor Theory: http://universe-review.ca/R15-19-twistor.htm Penrose R (2006). Before the Big Bang: An Outrageous New Perspective and its Implications for Particle Physics". Proceedings of the EPAC 2006, Edinburgh, Scotland: 27592762 Penrose R (2010). Cycles of Time, An Extraordinary New View of the Universe. London: Bodley Head. Penrose, R. (1994). Shadows of the Mind, Oxford University Press Penrose R, (1989). The Emperor 's New Mind. Oxford University Press: 1-275. Penrose, Roger (2004). The Road to Reality. A Complete Guide to the Laws of the Universe, Jonathan Cape, London Pereira A (2003): The quantum mind/classical problem. Neuro Quantology, 1: 94- 118.Pereira A, Furlan FA (2007).

[40]. Biomolecular Information, Brain Activity And Cognitive Function. In: ARBS Annual Rev Of Biomdical Sciences, 9: 12-29. Persinger M Dotta, B, Saroka K \& Scott M, (2013). Congruence of energies for cerebral photon emissions, quantative EEG activities and $\sim 5 \mathrm{nT}$ changes in the proximal geomagnetic field support spin-based hypothesis of consciousness. Journal of Consciousness Exploration and Research, February vol. 4, issue 1, pp. 1 24 Pessa E and Vitiello G (2003). "Quantum noise, entanglement and chaos in the quantum field theory of mind/brain states". Mind and Matter 1: 59-79. Pezzulo G, Barsalou LW, Cangelosi A, Fischer MH, McRae K, Spivey MJ. (2011). The mechanics of embodiment: a dialog on embodiment and computational modeling. Front Psychol. 2011;.Philosophical Approach (eds Juan José Sanguineti, Ariberto Acerbi, José Angel Lombo)Piaget, J. (1956). The general problem of the psychobiological development of the child. Discussions on Child Development, 1956, 4, 3-27. Pizzi, R, Fantasia A, Gelain F, Rosetti D, Vescovi A (2004). In: Quantum Information and Computation II. Procedings of SPIE 5436, (ed.) Donkor, E.

[41]. Popper K.R and Eccles J C. (1977). The self and its brain. Springer International. Berlin, Heidelberg, London, New York.; pp 3-566. Pothos EM Busemayer J. R. (2011). Formalizing heuristics in decision making; a quantum probability perspective. Front Psychology;2: 289. doi: 10.3389/fpsyg.2011.1100289 Pribram K (1991). Brain and perception: holonomy and structure in figural processing. Hillsdale, NJ: Lawrence Erlbaum Associates. Pribram K $\mathrm{H}$ and EH Carlton, (1986). Holonomic brain theory in imaging and object perception. Acta Psychologica: 63: 175180. Primas H (2003). Time entanglement between mind and matter. Mind and Matter, 1: 81-119. Primas H (2009). Complementarity of mind and matter. in: Recasting Reality, Berlin. Springer, 171-209 Pusey MF, Barret J and Rudolph $T$ (2012). On the reality of the quantum state. Nature Physics, 8, pp 475-478. http://lanl.arxiv.org/abs/1111.3328 Radin DI and Nelson R (2006). Entangled Minds. Extrasensory experiences in the quantum reality. New York: Simon \& Schuster Radin, Dean. (1997).

[42]. The Conscious Universe. The Scientific Truth of Psychic Phenomena. New York: HarperEdge. Raichle, M. (1998). The neural correlates of consciousness. Phil. Trans. Royal Soc London B, 353, pp. 1889 - 901 Reiter G.F., Kolesnikov A.I.,. Paddison S.J,. Platzman P.M,. Moravsky A.P, Adams M.A et al (2011). Evidence of a new quantum state of nano-confined water. http://arxiv.org/abs/1101.4994 Rieper, E. Anders J.,. Vedral V (2011).Quantum entanglement between the electron clouds of nucleic acids in DNA. http://arxiv.org/abs/1006.4053 Ricciardi, L.M., and Umezawa, H. (1967). Brain and physics of many-body problems. Kybernetik 4, 44-48. Romijn, H. (1997). About the origin of consciousness. Proceedings Kon. Akad, 100, pp. 181-267 Romijn H. (2002). Are virtual photons the elementary carriers of consciousness? Journal of Consciousness Studies 9 (1):61-81 (2002) Rosa LP, Faber J. (2004). Quantum models of the mind: are they compatible with environmental decoherence.Phys Rev E Stat Nonlin Soft Matter Phys. Sep;70(3 Pt 1):031902 Sahu S, Ghosh S, Ghosh B, et al( 2013a). Atomic water channel controlling remarkable properties of a single brain microtubule: correlating single protein to its supramolecular assembly. Biosensors and Bioelectronics.;4 7:141-148 Sahu S, Ghosh S, Hirata K, Fujita D, Bandyopadhyay (2013 b). Multi-level memory-switching properties of a single brain microtubule. Applied Physics Letters. 102(12): 4 pages.123701Sakai \& Miyashita, (1994). 
[43]. Neuronal tuning to learned complex forms in vision. Neuroreport, 5, 829 Salari V, Tuszynski, M. Rahnama, G Bernroider. Plausibility of Quantum Coherent States in Biological Systems http://arxiv. org/ftp/arxiv/papers/1012/1012.3879.pdf Sarfatti J (2011).

[44]. Retrocausality and Signal Nonlocality in Consciousness and Cosmology. Journal of Cosmology, 14: 3-33 Scaruffi P (2006). The nature of consciousness. http://www.scaruffi.com/nature/consc3.html Scaruffi P (2001). A simple theory of consciousness, http://www.scaruffi.com/science/sweden.html Schacter et al (1998). Memory, consciousness and neuroimaging. Phil. Trans Royal Soc. London, 353, pp. 1861-78 Schafer L (2009). Quantum Reality and Evolution Theory. Journal of Cosmology 3: 547-557. Schempp W (2003). Replication and transcription processes in the molecular biology of gene expressions: control paradigms of the DNA quantum holographic information channel in nanobiotechnology. BioSystems 68: 119-145. Schrödinger E (1959). Mind and Matter. Cambridge: University Press. Schrödinger E, (1986). What is Life? 1944. Pribram and Carlton Schwartz JM, Stapp HP, Beauregard M. (2004). Quantum physics in neuroscience and psychology: a neurophysical model of mind-brain interaction. Philosophical Transactions R Soc B; 1598: 1-19. Searle J R, (1992).

[45]. The rediscovery of mind. MIT Press Cambridge, Mass 0-262-19321-3, 978-0-262-69154- 3270 pp Searle JR, (2000). Consciousness. Ann Rev Neurosci 23: 557-578. Searle JR, (2007). Dualism Revisited. J Physiology (Paris) 101: 168-178 Sevush S. (2006). Single-neuron theory of consciousness. J Theoretical Biology, 238, 704-725 Sheldrake R (2012). The Science Delusion. Freeing the spirit of inquiry. Coronet/Hodder\&Stoughton. London. Sheldrake R. (2005). Morphic Resonance and Morphic Fields An Introduction http://www.sheldrake.org/research/morphic-resonance/introduction, also in J. Noetic Sci. 4, 2010 http://noetic.org/noetic/issue-four-november-2010/morphic-fields-and-morphic-resonance/Shimony A. (2005). "Quantum Mechanics and the Philosophy of Alfred North Whitehead (review)." Transactions of the Charles S. Peirce Society: A Quarterly Journal in American Philosophy 41.3; 714-723. Sirag S P. Consciousness, a hyperspace view. http://www.williamjames.com /Theory/ Consciousness.pdf Skarda CA, Freeman WJ (1987). How brains make chaos in order to make sense of the world. Behavioral and Brain Sciences, 10:161-195. Skrbina D (2005). Panpsychism in the West, MIT Press, Cambridge, MA. Smolin L (2004). Atoms of Space and Time. Scientific. Am. Febr: 43-52. Smythies, J. (2003). Space, time \& consciousness. Journal of Consciousness Studies, 10, (3), pp. 47-56. Soon CS, Brass M, Heinze HJ, Haynes JD (2008).

[46]. Unconscious determinants of free decisions in the human brain. Nat Neurosci 11: 543-545. Spinoza B (1677/1985). Ethics, in The Collected Works of Spinoza, Princeton: Princeton. Spivey M J (2007). The continuity of mind. New York Oxford University Press . pp Stapp H P (2009). Mind, Matter and Quantum Mechanics, BerlinHeidelberg: Springer-Verlag. Stapp, Henry P. (1998a). Pragmatic approach to consciousness In:s Brain and Values ed. Karl Pribram Stapp, HP (2012). Reply to a critic: Mind efforts, quantum zeno effect and environemental decoherence. NeuroQuantology,10: 601-605 Stuart CIJM, Takahashi Y, Umezawa H. (1978).On the stability and non-local properties of memory. Journ. of Theor Biol 71: 605-618. Steck D. (2009). Passage through chaos, Nature 461-736. Sudbury T (1997), The Fastest Way From A to B, Nature, 390, 11th December, 551-552. Sun Y. Wang C. Dai J. (2010).

[47]. Biophotons as neural communication signals demonstrated by in situ biophoton autography. Photochem Photobiol. Sci. 9, 315-322.10.1039/b9pp00125e Susskind L (1994). The world as a hologram. arXiv.org > hep-th $>$ arXiv:hep-th/9409089v2. Sutcliffe, M. \& Scrutton, N. (2000) .Enzyme catalysis .Trends in biochemical science, 25, pp. 40 Yasue, K. (1988). Wave cybernetics. Physical Review A38, 2671-2673 Tarlaci S (2010). A Historical View of the Relation between Quantum Mechanics and the Brain. Neuro Quantology, 8: 120-136 ' $t$ Hooft G (2001). The Holographic Principle. Basics and Highlights: in Fundamental Physics The Subnuclear Series, Vol. 37; Zuchichi, A., Ed.; World Scientific Singapore; pp 72-100.ETTERS PUBLISHED ONLINE: 14 NOVEMBER 2010 | Tegmark M (2000)

[48]. Importance of quantum decoherence in brain processes. Phys Rev E Stat Phys Plasmas Fluids Relat Interdiscip Topics, 61: 4194-4206. Tegmark M (2008). The mathematical universe. Found. Phys. 38:101-150, arXiv: 0704.0646 [gr-qc] Thompson I (2000). Dualism: a Causal Correspondence Theory. http://www.newdualism.org/papers/I.Thompson/qme1h.htmlTipler F (1995). The Physics of Immortality: Moder $n$ Cosmology, God and the Resurrection of the Dead. New York: Anchor Ultimate Reality, Cambridge: Cambridge University Pres. Tononi G and Koch C (2008). The Neural Correlates of Consciousness. An Update. Ann. N.Y. Acad. Sci. 1124: 239-261Trueblood JS Busemeyer J.R. (2012). A quantum probability model of causal reasoning. Front. Psychology 3: 138. doi: 10.3389/fpsyg. 2012.00138. Turennout M, Hagoort P, Brown CM (1998). Brain activity during speaking: from syntax to phonology in 40 milliseconds. Science.; 280: 572-574Tsai, H.C.; Zhang, F. ; Adamantidis, A.; Stuber, G. D. et al. (2009).

[49]. "Phasic firing in dopaminergic neurons is sufficient for behavioral conditioning". Science324(5930): 1080-4 Bibcode2009Sci.324.1080T. doi:10.1126/science.1168878. PMID 19389999. Turin L. (1996). A spectroscopic mechanism for primary olfactory reception. Chem Senses, 21 (6) pp. 773-791Tuszynski JA. and Woolf N (2010).

[50]. The Path Ahead. Introductory chapter of: The Emerging Physics of Consciousness. Springer verlag http://www.newdualism.org/books/Tuszynski/Tuszynski-EPC-1_2006.pdf Tuszynski JA. and Woolf N (2010). The Emerging Physics of Consciousness. Springer verlag Ullman S (1991). Sequence seeking and counter streams: A model for information processing in the cortex. MIT artificial intelligence laboratory. http://dspace.mit.edu/bitstream/handle/1721.1/6574/AIM - 1311.pdf?sequence=2 Umezawa H (1993). Advanced Field Theory. Macro and Thermal physics. New York:The American Institute of Physics Press. Utts J and B D Josephson(1996).

[51]. THE PARANORMAL: THE EVIDENCE AND ITS IMPLICATIONS FOR CONSCIOUSNESS. http://www.tcm.phy.cam.ac.uk/ bdj10/psi/tucson.html Life (Perseus, 2000), 368 pp, ISBN 0-7382-0436-6. Vannini A, Di Corpo U (2008). Quantum Models of Consciousness. Quantum Biosystems; 1(2): 165-184. Varela, F.J. (1995). Resonant cell assemblies: a new approach to cognitive function and neural synchrony, Biological Research 28:81-95. Varela, FJ.; Thompson, E; Rosch, Elean( 1991). The embodied mind: Cognitive science and human experience. MIT Press, Cambridge, MA, USA. Vattay G, Kauffman S, Niiranen S (2012). Quantum biology on the edge of quantum chaos. arXiv:1202.6433 (or arXiv:1202.6433v1) Vedral V (2012). Information and physics. Information, 3, 219-223 Vedral V (2010). Decoding Reality, University Oxford Press, Oxford, U.K. Verlinde EP (2011). On the Origin of Gravity and the Laws of Newton. JHEP 04, 29. Verlinde EP (2011). On the Origin of Gravity and the Laws of Newton. JHEP 04, 29 Vitiello G (2001). My Double Unveiled. The Dissipative 
Quantum Model of Brain. Advances in Consciousness Research, 32, Amsterdam: John Benjamins. Vitiello G (2001). My Double Unveiled. The Dissipative Quantum Model of Brain. Advances in Consciousness Rese arch, 32, Amsterdam: John Benjamins. Vitiello, G. (1995). "Dissipation and memory capacity in the quantum brain model". International Journal of Modern Physics B 9, 973-989. Vitiello, G. (1995). Dissipation and memory capacity in the quantum brain model. International Journal of Modern Physics B 9, 973-989. Von Neumann J (1963). Collected Works of John von Neumann, Taub, A. H., ed., Pergamon Press. Walker EH (2000). The Physics of Consciousness: The Quantum Mind and the Meaning of the Wheeler J.A. (1990). Information, physics, quantum: the search for links. Complexity, Entropy and the Physics of Information. Zurek, W.H., Ed.; Addison-Wesley, Redwood City, 3-28.

[52]. Wheeler JA, (2002). Quotation from: Does the Universe Exist if We're Not Looking? Discover Magazine. Physics \&Math / Cosmology June. Wheeler, J. A.; Feynman, R. P. (1949). "Classical Electrodynamics in Terms of Direct Interparticle Action". Reviews of Modern Physics 21 (3): 425-4332. doi:10.1103/RevModPhys.21.425 Whitehead C.W.A. (1961).The Relevance of Whitehead, Chapter 1, London: Allen and Unwin. Wigner, E P, (1992).

[53]. As told to Szanton, Andrew The Recollections of Eugene P. Wigner. Plenum. ISBN 0-306- 44326-0. Wolf FA (2008). Is the Mind of God Found in Quantum Field Theory? http://www. fredalanwolf.com/myarticles/Quantum\%20Field\%20Theory.pdf Wolf FA (1996). The Spiritual Universe. How Quantum Physics Proves the Existence of the Soul. New York: Simon \& Schuster. Woolf NJ, Hameroff SR (2001) A quantum approach to visual consciousness. Trends Cogn Sciences 5: 472-478 Yasue K and Jibu M (1995).

[54]. Quantum brain dynamics and consciousness, John Benjamins Wigner, E. P. (1960). "The unreasonable effectiveness of mathematics in the natural sciences. Richard Courant lecture in mathematical sciences delivered at New York University, May 11, 1959". Communications on Pure and Applied Mathematics 13: 1-14. Zeilinger A (1999). A Foundational Principle for Quantum Mechanics. Foundations of Physics 29 (4), 63-143. Zeilinger A (2000). Quantum Teleportation.

[55]. Scientific Am. Febr. 8-16. Update 2003: http://www.univie.ac.at/qfp/publications3/pdffiles/2003-24. pdf Zeki \& Bartels (1998). The autonomy of the visual systems and the modularity of conscious vision. Philos Trans R Soc

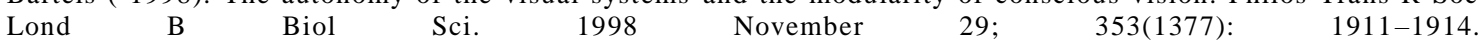
http://www.ncbi.nlm.nih.gov/pmc/articles/PMC1692424/Zohar D, Marshall I.N. (1990). The quantum self, human nature and consciousness defined by the new physics DKFM and SR, 12-3-2014Internetsites:Consciousness in general Thomas (basic quantum theory): http://www.ipod.org.uk/reality/CourseinConsciousness/Sobbotca:http://faculty.virginia.edu/consciousness/Course in Consciousness/Scaruffi: http://www.scaruffi.com/nature/index.htmlWebsiteMindPapers: http://consc.net/mindpapersWebsiteconsciousness:http://consc.net/online/8.8bIntegralconsciousness.,http://en.wiki pedia.org/wiki/Integral_thoughtWebsitesChalmers., http://consc.net/papers/moving.html. 